\title{
Line-profile variations due to adiabatic non-radial pulsations in rotating stars
}

\section{Observable characteristics of spheroidal modes}

\author{
C. Schrijvers ${ }^{1}$, J.H. Telting ${ }^{1}$, C. Aerts ${ }^{2, \star}$, E. Ruymaekers ${ }^{3}$, and H.F. Henrichs ${ }^{1}$ \\ 1 Astronomical Institute Anton Pannekoek, University of Amsterdam, and Center for High Energy Astrophysics, Kruislaan \\ 403, 1098 SJ Amsterdam, The Netherlands \\ 2 Instituut voor Sterrenkunde, Katholieke Universiteit Leuven, Celestijnenlaan 200 B, B-3001 Heverlee, Belgium \\ 3 St. Antoniusstraat 139, B-8500 Kortrijk, Belgium
}

Received February 29; accepted June 3, 1996

\begin{abstract}
We present a useful formulation of the surfacevelocity field of a rotating, adiabatically pulsating star, which accounts for the effects of the Coriolis force. We use this model to investigate the observable spectroscopic characteristics of non-radial pulsations. We calculate time series of absorption line profiles in a carefully chosen domain of parameter space. Only mono-periodic spheroidal modes are investigated; atmospheric changes due to the pulsation are neglected. The line-profile variations, as well as their behavior inferred from two well-defined diagnostics, are presented in two-dimensional parameter grids. We show that the intensity variations in time series of theoretical spectra, at each position in the line profile, cannot be described by a single sinusoid: at least one harmonic sinusoid needs to be included. Across the line profile the relative amplitudes and phases of these sinusoids vary independently. The blue-to-red phase difference found at the main pulsation frequency turns out to be an indicator of the degree $\ell$, rather than the azimuthal order $|m|$; the phase difference of the variations with the first harmonic frequency is an indicator of $|m|$. Hence, the evaluation of the variability at the harmonic frequency can improve the results derived from an analysis of observed line profiles. We find, that if line-profile variations at the line center dominate over the variations in the line wings, this does not give conclusive information on the ratio of the horizontal to the vertical pulsational surface motions. Tesseral modes, when observed at not too high inclinations, are as much capable of producing considerable line-profile variations as sectoral modes. We find that, within the limits of our model, the effects of rotation on the appearance
\end{abstract}

Send offprint requests to: Coen Schrijvers, Internet: coen@astro.uva.nl

* Senior Research Assistant, Belgian National Fund for Scientific Research. of the line-profile variations are important for low-degree sectoral modes, and for the sub-class of the tesseral modes with $\ell-m$ an even number.

Key words: line: profiles — stars: oscillations, rotation - stars: variable: $\delta$ Sct stars; others

\section{Introduction}

The study of stellar pulsations can improve our understanding of stellar structure. By measuring the photometric and spectral line-profile variations caused by pulsations, one can determine the time dependent state of the stellar surface, and eventually probe the interior of the star. The development of high-precision spectroscopy with solid-state detectors initiated the discovery of several classes of non-radially pulsating early-type stars (e.g., Smith 1977; Vogt \& Penrod 1983; Baade 1984; Gies \& Kullavanijaya 1988), and offered much higher accuracy in measuring time variability of line profiles. The increase in computing power allowed the numerical synthesis of lineprofile variations, based on analytical descriptions of the surface velocity and/or temperature perturbations due to non-radial pulsations (Osaki 1971; Kambe \& Osaki 1988; Lee \& Saio 1990; Aerts \& Waelkens 1993). Such numerical simulations are indispensable for the correct interpretation of observed line-profile variability. An excellent textbook on the theory of stellar pulsations is written by Unno et al. (1989).

Spectroscopic identifications of pulsation modes have been attempted by fitting theoretical line profiles to observed spectra (e.g. Smith 1977). A serious problem with such an approach is the large number of free parameters, 
and the associated question of the uniqueness of the solution. Currently, two powerful Fourier-analysis techniques are in use, which attempt to resolve this problem. The first is based on the Doppler imaging principle (Vogt et al. 1987), in which one assumes a mapping of photospheric features (e.g. local velocity, brightness or EW variations) onto line profiles that are Doppler broadened by the rotation of the star. From a Fourier transform applied to each velocity bin of a time series of observed spectra one obtains the power of variability as a function of frequency (periodogram), for all velocity positions in the line profile (e.g. Gies \& Kullavanijaya 1988; Kambe et al. 1990; Reid et al. 1993; see Kennelly et al. 1992 for an alternative version of this technique which comprises a 2D Fourier transform). Additionally, the Fourier analysis provides information about the phase change of the periodic variations across the line profile. Using the power and phase information, some of the pulsation parameters can be derived.

The second technique involves the computation of velocity moments of the line profile (Balona 1986; Aerts et al. 1992). A comparison of the Fourier components derived from the observed moment variations with those of the velocity moments generated by means of a non-radial pulsation model, gives in principle all the desired pulsation parameters.

In this paper we apply a technique, that is similar to the first mentioned method, to theoretical spectra. Our aim is to illustrate the dependence of the observable diagnostics (which will be defined in Sect. 4) on the relevant stellar and pulsational parameters. To allow a comparison with the work of Aerts et al. (1992), we calculated the first two velocity moments as well. We present calculations for rotating early-type stars, but in many cases our description applies to other types of stars as well.

We use a description of the pulsational velocity field of a rotating star that incorporates terms due to the Coriolis force, which is equivalent to the one presented by Aerts \& Waelkens (1993, hereafter AW), but with a few modifications to allow straightforward calculation. We investigate only single spheroidal pulsation modes. A generalization of the velocity field to multiple modes in a rotating star is straightforward if a linear pulsation theory applies. In all our models, we assume the symmetry axis of the pulsation to be aligned with the axis of rotation. In our line profile synthesis we neglect the atmospheric variations due to the pulsation, which is justified for those absorption lines for which the pulsational velocity variations dominate the temperature effects (Simon 1991).

In subsequent papers we present the results of bulk computations of the diagnostics (Paper II, Telting \& Schrijvers 1996a), we discuss the peculiar line-profile behavior of nearly equator-on tesseral modes with odd $\ell-m$ (Paper III, Telting \& Schrijvers 1996b), and we investigate the effect of brightness and EW changes caused by the pulsation on the observational diagnostics (Paper IV,
Schrijvers \& Telting 1996). Preliminary results of the diagnostic value of phase diagrams derived from time series of spectra of non-radially oscillating stars are presented by Telting \& Schrijvers (1995).

The outline of this paper is as follows. In Sects. 2.1 and 2.2 we briefly summarize the traditional model describing the surface velocity field of non-radial adiabatic pulsations, in the limit of no rotation. The effect of rotation on the velocity field of pulsation is included in Sect. 2.3. In Sect. 2.4 we derive an expression for the ratio of the horizontal to the vertical pulsational amplitude, $k$, which accounts for effects of the Coriolis force, and in Sect. 2.5 we verify this expression in the case of a polytropic model. The synthesis of line profiles is described in Sect. 3. We discuss the observable diagnostics in Sect. 4 . The relevant domains of the pulsational and stellar parameters are discussed in Sect. 5. The results of our computations are presented in Sect. 6. We summarize our findings in Sect. 7.

\section{Surface velocity field of adiabatic pulsations}

We first briefly summarize the traditional expression for the surface velocity field of non-radial pulsations in the limit of no rotation. We will refer to this as the zerorotation model. Subsequently, we give a more general description which accounts for the effects of the Coriolis force on the displacement field of the pulsation. This description, which we refer to as the slow-rotation model, is equivalent to those of Saio (1981), Martens \& Smeyers (1982) and Aerts \& Waelkens (1993). Hereafter we will refer to these three papers jointly as SMA.

\subsection{Basic equations}

The equations that govern linear, isentropic pulsations of a rotating star are the equations of motion, continuity, conservation of entropy, and Poisson's equation. In our treatment the distortion of the spherical equilibrium surface by centrifugal forces is neglected, as well as the effects of centrifugal forces on the pulsation (which are proportional to $\Omega^{2}$, with $\Omega$ the angular rotation frequency of the star): only the effects of the Coriolis force $(\propto \Omega)$ are taken into account. Therefore, our description is only valid for slowly rotating stars. We will quantify this restriction in Sect. 5.1. Following SMA, we assume that the evolution of the star is a succession of quasi-static states of hydrostatic and thermal equilibrium, and that the temporal part of the pulsation can be written as $\mathrm{e}^{i \omega t}$, where $\omega$ is the angular frequency of pulsation in the corotating frame and $t$ is time. (Hereafter, we speak of frequencies when we mean angular frequencies.) We write the perturbed and linearized equations as

$$
\begin{aligned}
& \omega^{2} \boldsymbol{\xi}+2 i \omega(\boldsymbol{\Omega} \times \boldsymbol{\xi})=\nabla \Phi^{\prime}-\frac{\rho^{\prime}}{\rho^{2}} \nabla P+\frac{1}{\rho} \boldsymbol{\nabla} P^{\prime} \\
& \frac{\rho^{\prime}}{\rho}+\frac{1}{\rho} \frac{\mathrm{d} \rho}{\mathrm{d} r} \xi_{r}+\boldsymbol{\nabla} \cdot \boldsymbol{\xi}=0
\end{aligned}
$$


$\frac{\rho^{\prime}}{\rho}+\frac{1}{\rho} \frac{\mathrm{d} \rho}{\mathrm{d} r} \xi_{r}=\frac{1}{\Gamma_{1}}\left(\frac{P^{\prime}}{P}+\frac{1}{P} \frac{\mathrm{d} P}{\mathrm{~d} r} \xi_{r}\right)$

$\nabla^{2} \Phi^{\prime}=4 \pi G \rho^{\prime}$

in which $\boldsymbol{\xi} \equiv\left(\xi_{r}, \xi_{\theta}, \xi_{\phi}\right)$ is the Lagrangian displacement vector, $\rho, P$ and $\Phi$ are the mass density, the pressure, and the gravitational potential, $\rho^{\prime}, P^{\prime}$, and $\Phi^{\prime}$ are their Eulerian perturbations, $i=\sqrt{-1}$, and $\Gamma_{1}$ is the first generalized isentropic coefficient. This set of six equations determines the state of a rotating, pulsating star.

\subsection{Description in the limit of no rotation}

Since our aim is to calculate line-profile variations, we need to describe the pulsational velocity field at the surface of the star. Neglect of the term proportional to $\Omega$ in Eq. (1) leads to the well-known solution of the perturbation problem for which the angular dependence of the displacement field of a normal mode is specified by one spherical harmonic $Y_{\ell}^{m}(\theta, \phi)$ and its derivatives. The spherical harmonic describes the shape of the perturbation as a function of co-latitude $\theta$ measured from the polar axis, and azimuth $\phi$. We write the spherical harmonics as

$Y_{\ell}^{m}(\theta, \phi) \equiv P_{\ell}^{|m|}(\cos \theta) \mathrm{e}^{i m \phi}$,

and the Associated Legendre polynomial $P_{\ell}^{m}(x)$ as

$P_{\ell}^{m}(x) \equiv \frac{(-1)^{m}}{2^{\ell} \ell !}\left(1-x^{2}\right)^{m / 2} \frac{\mathrm{d}^{\ell+m}}{\mathrm{~d} x^{\ell+m}}\left(x^{2}-1\right)^{\ell}$,

where $\ell$ and $m=-\ell, \ldots,+\ell$ denote the degree and azimuthal order of the mode respectively. The Lagrangian displacement vector for spheroidal modes of a non-rotating star in spherical coordinates is then given by

$\boldsymbol{\xi}=\left(a^{(0)}, b^{(0)} \frac{\partial}{\partial \theta}, b^{(0)} \frac{1}{\sin \theta} \frac{\partial}{\partial \phi}\right) N_{\ell}^{m} Y_{\ell}^{m}(\theta, \phi) \mathrm{e}^{i \omega^{(0)} t}$,

with $a^{(0)}$ and $b^{(0)}$ the vertical and horizontal displacement amplitudes. The superscripts ${ }^{(0)}$ refer to quantities in the non-rotating case. $N_{\ell}^{m}$ is a normalization constant

$N_{\ell}^{m}=(-1)^{\frac{m+|m|}{2}} \sqrt{\frac{2 \ell+1}{4 \pi} \frac{(\ell-|m|) !}{(\ell+|m|) !}}$.

Note that our definition of $N_{\ell}^{m}$ differs from those of SMA and Unno et al. (1989), but that this difference is not important for our computations of the line-profile variability since we use the maximum surface velocity to parameterize the pulsation amplitude (see Sect. 5.4).

The useful parameter $k$ is defined as the ratio of the horizontal to the vertical amplitude. In the Cowling approximation $\left(\Phi^{\prime}=0\right), k$ can be evaluated at the stellar surface by

$k^{(0)} \equiv \frac{b^{(0)}}{a^{(0)}}=\frac{G M}{\left(\omega^{(0)}\right)^{2} R^{3}}$,

with $G, M$ and $R$ the gravitational constant, the stellar mass and radius, respectively. We discuss the validity of the Cowling approximation for the determination of $k$ in Sect. 2.5.

The surface velocity field in the corotating frame is then completely specified by $\ell, m, a^{(0)}, k^{(0)}$, and $\omega^{(0)}$. We refer to this description as the zero-rotation model. Note that this does not mean that the star does not rotate, but rather that we are using the zero-rotation approximations to describe its pulsational behavior.

\subsection{Velocity field of adiabatic pulsations of a rotating star}

We now consider the effects of the Coriolis force on the properties of the pulsation of a rotating star. Following SMA we expand all unknown quantities in a zero-rotation part and a correction term due to the Coriolis force. The correction terms are proportional to the parameter $\Omega / \omega^{(0)}$ (which is assumed to be smaller than unity, see below). The vertical and horizontal Lagrangian displacement amplitudes $a$ and $b$ become

$a \equiv a^{(0)}+\frac{\Omega}{\omega^{(0)}} a^{(1)}, \quad b \equiv b^{(0)}+\frac{\Omega}{\omega^{(0)}} b^{(1)}$,

and similarly, the pulsation frequency is written as (in analogy with Ledoux 1951)

$\omega \equiv \omega^{(0)}+\frac{\Omega}{\omega^{(0)}} \omega^{(1)}=\omega^{(0)}+m \Omega C_{n \ell}$,

which describes the frequency splitting caused by the rotation of the star. The constant $C_{n \ell}$ (Ledoux 1951; Hansen et al. 1978) depends on the internal structure of the star, and on the degree $\ell$ and radial order $n$ of the mode, and hence $C_{n \ell}$ contains asteroseismological information.

Since the effects of the centrifugal force $\left(\propto\left(\Omega / \omega^{(0)}\right)^{2}\right)$ are neglected, the expansions above are, in general, only applicable to cases where $\Omega / \omega^{(0)} \ll 1$. Substituting expansions similar to those in Eqs. (10) and (11) for all quantities in the set of Eqs. (1-4), and neglecting all terms of the second and higher orders in $\Omega / \omega^{(0)}$, leads to a new system of equations which needs to be solved to determine the unknown first-order quantities.

As shown by SMA, the eigenfunctions of the star can now be written as a superposition of the zero-rotation eigenfunctions (Eq. 7) and one spheroidal and two toroidal terms. The three latter terms are due to the Coriolis force. We express the Lagrangian displacement field at the surface of a rotating star by

$$
\begin{gathered}
\boldsymbol{\xi}=a_{\mathrm{sph}, \ell}\left(1, k \frac{\partial}{\partial \theta}, k \frac{1}{\sin \theta} \frac{\partial}{\partial \phi}\right) N_{\ell}^{m} Y_{\ell}^{m}(\theta, \phi) \mathrm{e}^{i \omega t} \\
+a_{\mathrm{tor}, \ell+1}\left(0, \frac{1}{\sin \theta} \frac{\partial}{\partial \phi},-\frac{\partial}{\partial \theta}\right) N_{\ell}^{m} Y_{\ell+1}^{m}(\theta, \phi) \mathrm{e}^{i\left(\omega t+\frac{\pi}{2}\right)} \\
+a_{\mathrm{tor}, \ell-1}\left(0, \frac{1}{\sin \theta} \frac{\partial}{\partial \phi},-\frac{\partial}{\partial \theta}\right) N_{\ell}^{m} Y_{\ell-1}^{m}(\theta, \phi) \mathrm{e}^{i\left(\omega t-\frac{\pi}{2}\right)}
\end{gathered}
$$


in which the zeroth and first order spheroidal terms are combined in the first term. We note that, instead of $N_{\ell}^{m}$, AW incorrectly use $N_{\ell+1}^{m}$ and $N_{\ell-1}^{m}$ to normalize the toroidal terms in their Eq. (19), and that their implementation of the velocity field contains a small error (Aerts $\&$ Waelkens 1995). With our definition of $Y_{\ell}^{m}$, the amplitudes $a_{\mathrm{sph}, \ell}, a_{\mathrm{tor}, \ell+1}$, and $a_{\mathrm{tor}, \ell-1}$ in Eq. (12) are given by

$a_{\mathrm{sph}, \ell}=a^{(0)}+\frac{\Omega}{\omega^{(0)}} a^{(1)}$,

$a_{\mathrm{tor}, \ell+1}=\frac{\Omega}{\omega^{(0)}} \frac{\ell-|m|+1}{\ell+1} \frac{2}{2 \ell+1}\left(1-\ell k^{(0)}\right) a^{(0)}$,

$a_{\mathrm{tor}, \ell-1}=\frac{\Omega}{\omega^{(0)}} \frac{\ell+|m|}{\ell} \frac{2}{2 \ell+1}\left(1+(\ell+1) k^{(0)}\right) a^{(0)}$.

Note that for sectoral $(\ell=|m|)$ and radial $(\ell=0)$ modes the term proportional to $Y_{\ell-1}^{m}$ in Eq. (12) equals zero.

For a given stellar model, the amplitude of the firstorder spheroidal correction $a^{(1)}$ in Eq. (13) can be determined from the zeroth-order solution of the pulsation mode. In our general treatment we avoid the introduction of an additional parameter $a^{(1)}$, making use of an approximation that is allowed in a first-order approach: instead of $a^{(0)}$, we substitute $a_{\mathrm{sph}, \ell}$ into Eqs. (14) and (15), which introduces an error of the second order in $\Omega / \omega^{(0)}$ in Eq. (12). This substitution allows us to study the effect of the toroidal terms, which arise as a consequence of rotation. The effect of rotation is then determined only by the rotation parameter $\Omega / \omega^{(0)}$, which becomes the sixth parameter of the surface velocity field in addition to the five mentioned above: $\ell, m, a_{\mathrm{sph}}, k^{(0)}, \omega^{(0)}$, and $\Omega / \omega^{(0)}$. In the following, we refer to this description as the slow-rotation model.

\subsection{An expression for $k$, correct to the first order in $\Omega / \omega^{(0)}$}

In the slow-rotation model, $k$ is modified by rotation and may differ significantly from Eq. (9). AW give an expression for $k$ which is correct up to the first order in $\Omega / \omega^{(0)}$, but their result contains the unknown parameters $a^{(1)}$ and $b^{(1)}$ and therefore cannot be used in applications. We derive a new expression to approximate $k$, up to the first order in $\Omega / \omega^{(0)}$, from the boundary condition

$\delta P=P^{\prime}+\boldsymbol{\xi} \cdot \nabla P=0$,

at the surface of the star. Our derivation of $k$ is similar to the one in the zero-rotation model. Under the assumptions of spherical symmetry and hydrostatic equilibrium, the condition (16) leads to

$\frac{P_{\ell}^{\prime(0)}}{\rho R}+\frac{\Omega}{\omega^{(0)}} \frac{P_{\ell m}^{\prime(1)}}{\rho R}=\frac{G M}{R^{3}}\left(a^{(0)}+\frac{\Omega}{\omega^{(0)}} a^{(1)}\right)$.
From the $\phi$-component of the equation of motion of order zero in $\Omega / \omega^{(0)}$ (Eq. 1), we find that

$\frac{P_{\ell}^{\prime(0)}}{\rho R} \approx \frac{1}{R}\left(\frac{P_{\ell}^{\prime(0)}}{\rho}+\Phi_{\ell}^{\prime(0)}\right)=\left(\omega^{(0)}\right)^{2} b^{(0)}$.

Similarly, an expression for $P_{\ell m}^{\prime(1)}$ is given by

$$
\begin{aligned}
\frac{P_{\ell m}^{\prime(1)}}{\rho R} & \approx \frac{1}{R}\left(\frac{P_{\ell m}^{\prime(1)}}{\rho}+\Phi_{\ell m}^{\prime(1)}\right) \\
& =\left(\omega^{(0)}\right)^{2}\left(b^{(1)}+2 m C_{n \ell} b^{(0)}-\frac{2 m\left(a^{(0)}+b^{(0)}\right)}{\ell(\ell+1)}\right)
\end{aligned}
$$

(see e.g. Eq. (13) in AW). Note that we used the Cowling approximation $\left(\Phi^{\prime}=0\right)$ in both Eq. (18) and (19). We use Eqs. $(10-11,17-19)$, and neglect terms of order $\left(\Omega / \omega^{(0)}\right)^{2}$ to obtain

$b=\frac{G M}{\omega^{2} R^{3}} a+\frac{2 m}{\ell(\ell+1)} \frac{\Omega}{\omega}(a+b)$.

This leads to the following expression for $k$, correct to the first order in the parameter $\Omega / \omega^{(0)}$

$k \equiv \frac{b}{a}=\frac{G M}{\omega^{2} R^{3}}+\frac{2 m}{\ell(\ell+1)} \frac{\Omega}{\omega}\left(1+\frac{G M}{\omega^{2} R^{3}}\right)$.

Relating $k$ to the zero-rotation frequency $\omega^{(0)}$ rather than to the pulsation frequency $\omega$ leads to the desired expression, correct up to the first order in $\Omega / \omega^{(0)}$

$k \equiv \frac{b}{a}=k^{(0)}+2 m \frac{\Omega}{\omega^{(0)}}\left(\frac{1+k^{(0)}}{\ell(\ell+1)}-C_{n \ell} k^{(0)}\right)$,

in which we used Eqs. (9) and (11).

Equation (22) shows that the structure constant $C_{n \ell}$ enters the description of the surface-velocity field, when first-order terms due to the rotation are included. In the case of $p$-modes with high radial order, $C_{n \ell}$ tends to zero, while for $g$-modes with high radial order it tends to $1 / \ell(\ell+1)$ (Unno et al. 1989). For high-order $p$-modes, the term with $C_{n \ell}$ in Eq. $(22)$ vanishes $\left(k^{(0)} \rightarrow 0\right)$. On the other hand, as we will discuss in Sect.5.6, for large $k^{(0)}$ values (i.e. high-order $g$-modes) a small change in $k$ does not change the characteristics of the line-profile variations. Hence, for these two limiting cases of high order $p$ - and $g$ modes, the line profiles do not depend on the actual value for $C_{n \ell}$. For cases with intermediate $k^{(0)}$-values, $C_{n \ell}$ codetermines the line profiles via the first-order correction of $k$. In the slow-rotation model, we assume $C_{n \ell}=1 / 2 \ell(\ell+1)$ for simplicity, which is in between the two limiting cases for $p$ - and $g$-modes of high radial order. This approximation might not be accurate for fundamental modes. For numerical calculations of $C_{n \ell}$ we refer to Hansen et al. (1978) and to Carroll \& Hansen (1982).

By means of Eq. (22), we draw the following conclusions about the correction of $k^{(0)}$ by the rotation of the 
star. In the case of high-order $g$-modes (large $\left.k^{(0)}\right)$, the first-order correction term for $k$ is smaller than the zerothorder component if $\Omega / \omega^{(0)}<0.5$. This means that for these modes horizontal motions dominate like in the nonrotating case. For $p$-modes (i.e. low $k^{(0)}$ ), however, the first-order correction term of $k$ may become larger than the zeroth-order term, reflecting the fact that the rotation can reduce radial motion in favor of horizontal motion. For certain prograde modes $(m<0)$, this could theoretically give rise to negative $k$-values. However, as will be discussed in Sect. 5.1, for the small $k^{(0)}$-values associated with $p$-modes, the relative rotation rate $\Omega / \omega^{(0)}$ is also small. Therefore, the small $k^{(0)}$-values associated with $p$ modes will never be corrected by the rotation in such a way that a large negative $k$-value is found. We note that the first-order correction of $k$ is most important for modes of low degree $\ell$.

\subsection{Verification of the derived expression for $k$ for $a$ polytropic model}

Here we address the question: how large is the error in $k$ due to the Cowling approximation? For this purpose, we have used the computer code by De Boeck (De Boeck, in preparation) to determine the ratio of the amplitudes $b$ and $a$ by means of an approach that does not make use of the Cowling approximation. The code performs a numerical integration of a system of differential equations describing the pulsations up to first order in $\Omega / \omega^{(0)}$ in the case of a polytropic model with index 3 . The numerical values for $k$ obtained in this way were then compared with the ones obtained from Eq. (22), for both $p$ - and $g$-modes $\left(p_{1}, \ldots, p_{5}\right.$ and $\left.g_{1}, \ldots, g_{10}\right)$. For each mode we have considered $\ell=0, \ldots, 5$, with $m=-\ell, \ldots, \ell$, and $\Omega / \omega^{(0)}=0.0,0.1,0.2,0.3,0.4,0.5$. In all the considered cases, the two $k$-values differ less than $5 \%$.

To derive the $k$-value for a real star, we need to know its mass, radius, and pulsation frequency in the corotating frame. Since in practice the uncertainty in $M / R^{3}$ is likely to be larger than $5 \%$, we conclude that the error in $k$ due to the Cowling approximation is much smaller than that due to the inaccuracy of the stellar parameters. The expression for $k$ is therefore accurate enough for our purposes.

\section{Line-profile synthesis}

The velocity field of pulsation, described in the corotating frame, is given by the time derivative of the Lagrangian displacement field

$\boldsymbol{V}=\frac{\partial \boldsymbol{\xi}}{\partial t}=i \omega \boldsymbol{\xi}$

which we calculate on a spherical grid of typically more than 5000 equally sized visible surface elements. For each visible element, the corresponding rotational velocity of a uniformly rotating sphere is added. Neglecting all atmospheric effects due to the pulsation, we attribute to each surface element a Gaussian intrinsic line profile with width W

$f_{\text {intr }}(V)=1-\frac{d_{\text {intr }}}{W \sqrt{\pi}} \mathrm{e}^{-\left(\left(V-V_{0}\right) / W\right)^{2}}$,

where the free parameter $d_{\text {intr }}$ is a measure of the line depth and $V_{0}$ the central velocity position of the Dopplershifted line profile of the regarded surface element. The line profile $I(V)$ is then obtained by a weighted integration of the Doppler-shifted Gaussian profiles of all visible surface elements. Finally, the profile $I(V)$ is normalized by means of a division by its continuum value.

We note that our conclusions will not depend on the actual shape of the intrinsic line profile. The difference between profiles calculated with either a Gaussian or, for example, a rectangular shape is only noticeable in the wings of the rotationally broadened line profile. If the rotational broadening is large enough (i.e. $V_{\mathrm{e}} \sin i>W$ ), only the effective width of the intrinsic line profile is important.

The weights in the integration are determined by the aspect angle of each element, neglecting the rotational and pulsational deformation of the stellar surface, and by a linear limb-darkening correction of the form

$\frac{I(\vartheta)}{I(0)}=1-\alpha+\alpha \cos \vartheta$

in which $\vartheta$ is the angle between the surface normal of the sphere and the line of sight. We adopt a limb-darkening coefficient $\alpha=0.35$, which is appropriate for early B-type stars in the optical region (Wade \& Rucinski 1985). For simplicity we apply the same limb-darkening correction to the continuum and to the wavelength region of the intrinsic line profile itself. This means that we keep the shape of the intrinsic profile constant over the stellar disk. For very strong lines this might not be a good approximation.

We checked whether or not the use of other values of the limb-darkening coefficient $\alpha$ affects the validity of any of the conclusions in this paper. The phases of variability across the profiles do not change, but the amplitude distribution (see Sect.4) does. In our representation of the amplitudes of line-profile variability (see Sect. 4.1), a high limb-darkening coefficient results in higher variational amplitudes at the line center, and lower amplitudes at the wings of the profile. A limb-darkening coefficient of 1.0 can lead to changes up to $20 \%$ of the variational amplitudes with respect to the case with no limb darkening. Nevertheless, our conclusions do not depend on our choice for the limb-darkening coefficient.

In this work we aim to investigate the observational characteristics of line-profile variations in the best situation one can consider, namely that of perfect data. Following this approach, we can establish which pulsation parameters one may ultimately derive from observations. For this reason, we generate data that perfectly sample the pulsation period, with sufficient spectral 
resolution, and we do not include noise in the synthesis of our spectra.

In the case of real data, the data sampling and data quality (e.g. signal to noise ratio, $\mathrm{S} / \mathrm{N}$, spectral resolution, and correct continuum rectification) govern the detectability of the pulsation characteristics. The interplay between the time sampling and the $\mathrm{S} / \mathrm{N}$ is complicated and may hamper the detection of characteristics of certain pulsation modes in a complex fashion. Generally, we expect that from lengthy data sets the most conclusive results can be derived.

\section{Diagnostic tools for the analysis of line-profile variability}

In practice one aims to derive pulsation parameters from observed spectra. To enable a comparison of our results with that of observational studies, we analyze the artificial time series of spectra with two diagnostic methods, each closely related to techniques discussed in the literature (Gies \& Kullavanijaya 1988; Aerts et al. 1992).

\subsection{Morphology of amplitude- and phase distribution across the line profile}

We calculated amplitude and phase diagrams as diagnostic tools for studying line-profile variations. The diagrams are equivalent to the power and phase diagrams obtained by the popular method of Fourier analysis proposed by Gies \& Kullavanijaya (1988).

The apparent frequency of observed line-profile variability $\left(\omega_{\text {obs }}\right)$ has to be determined by Fourier techniques. For synthetic line profiles it is equal to the input frequency of the variations. Since the intensity variations in the line profile are not strictly sinusoidal (see Fig. $8 \mathrm{~b}$ in Gies 1991), there will be also some fraction of the total variational power distributed over harmonics. Once the main frequency of the line-profile variation is known, the variability of the normalized intensity can be decomposed into its harmonic contributions, which is in our case conveniently achieved by fitting a combination of sinusoids of the form

$$
\begin{aligned}
\Delta I(V, t) & =I_{0}(V) \sin \left(\omega_{\text {obs }} t+\Psi_{0}(V)\right) \\
& +I_{1}(V) \sin \left(2 \omega_{\text {obs }} t+\Psi_{1}(V)\right) \\
& +I_{2}(V) \sin \left(3 \omega_{\text {obs }} t+\Psi_{2}(V)\right)
\end{aligned}
$$

to the intensity variations of a time series of generated spectra. In general, the amplitudes of the intensity variations decrease for higher harmonics. Harmonics higher than the second $\left(3 \omega_{\text {obs }}\right)$ are not taken into account in the fits since their amplitudes are too small to be of importance in practice.

In Fig. 1 we illustrate the importance of harmonics in the analysis line profile variations. It appears that in general at least one harmonic is needed to properly describe
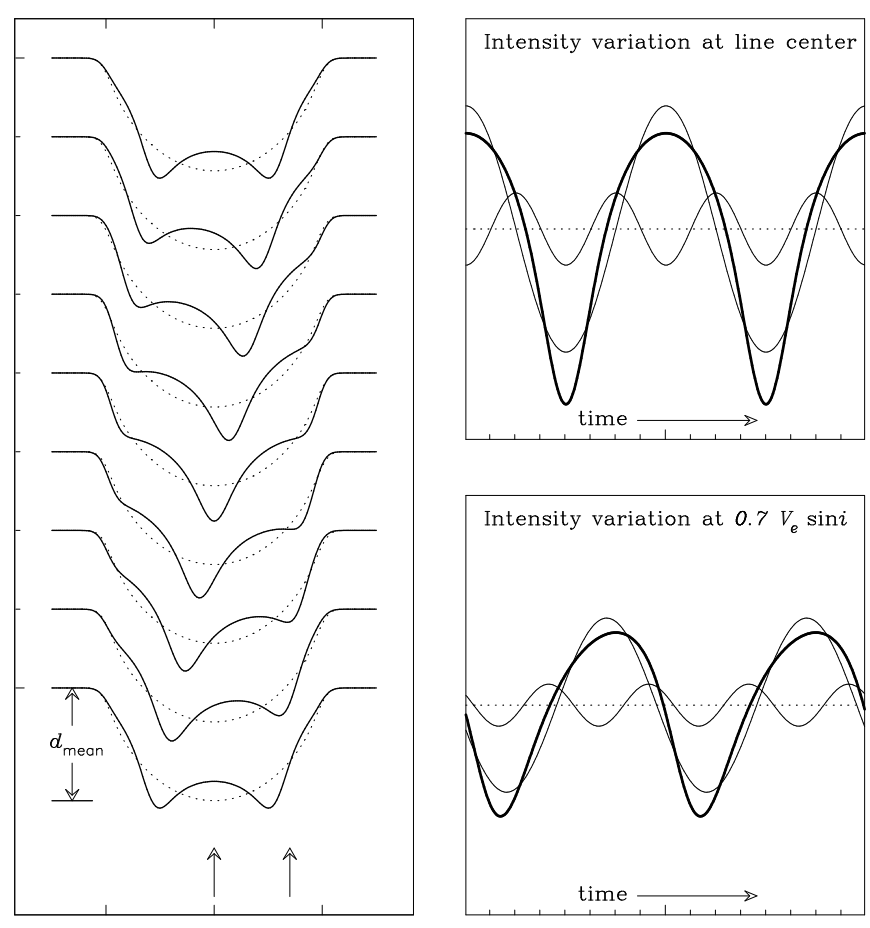

Fig. 1. Line-profile variability for a pulsation mode with input parameters $\ell=5, m=-5, V_{\max }=0.1 V_{\mathrm{e}} \sin i, k^{(0)}=0.0, W$ $=0.1 V_{\mathrm{e}} \sin i, i=75^{\circ}$, and $\Omega / \omega^{(0)}=0.0$ (zero-rotation model). Left: Line profiles during one pulsation cycle, with time increasing upwards. The first and the last profile are identical. The dotted line indicates the mean of the time series. The separation between the tick marks at the velocity axis is $V_{\mathrm{e}} \sin i$. The two arrows at the bottom refer to $V=0$ and $V=0.7 V_{\mathrm{e}} \sin i$. Right: Top: Intensity variations in line center $(V=0)$. The variations are shown during two pulsation cycles. The time tick marks correspond to those at the vertical axis of the left plot. The intensity variation is indicated by a thick line and is measured with respect to the mean profile. The thin lines show the decomposition of the signal into sinusoids with once and twice the apparent frequency. Bottom: Same as top panel but at velocity $V=0.7 V_{\mathrm{e}} \sin i$. The intensity scales are equal for top and bottom panel

the variability. The phases of the sinusoids, as a function of position in the line profile, describe the motion of the bumps that cross the profile. Through the line profile the phases and amplitudes of the sinusoids show independent behavior, which is a clear indication that the inclusion of a harmonic provides additional information to the analysis of the line-profile variability. Taking into account one or more harmonics can therefore improve the identification of pulsation parameters from observed spectra.

For the apparent frequency and its first two harmonics, we calculate the amplitudes $\left(I_{0}, I_{1}, I_{2}\right)$ and phases $\left(\Psi_{0}, \Psi_{1}, \Psi_{2}\right)$ for each position in the line profile. We remove the $2 \pi$ wrap from $\Psi_{0}, \Psi_{1}$ and $\Psi_{2}$ to create continuous blue-to-red phase diagrams. In the figures which result from our grid calculations (Figs. 3-9) we only plot 

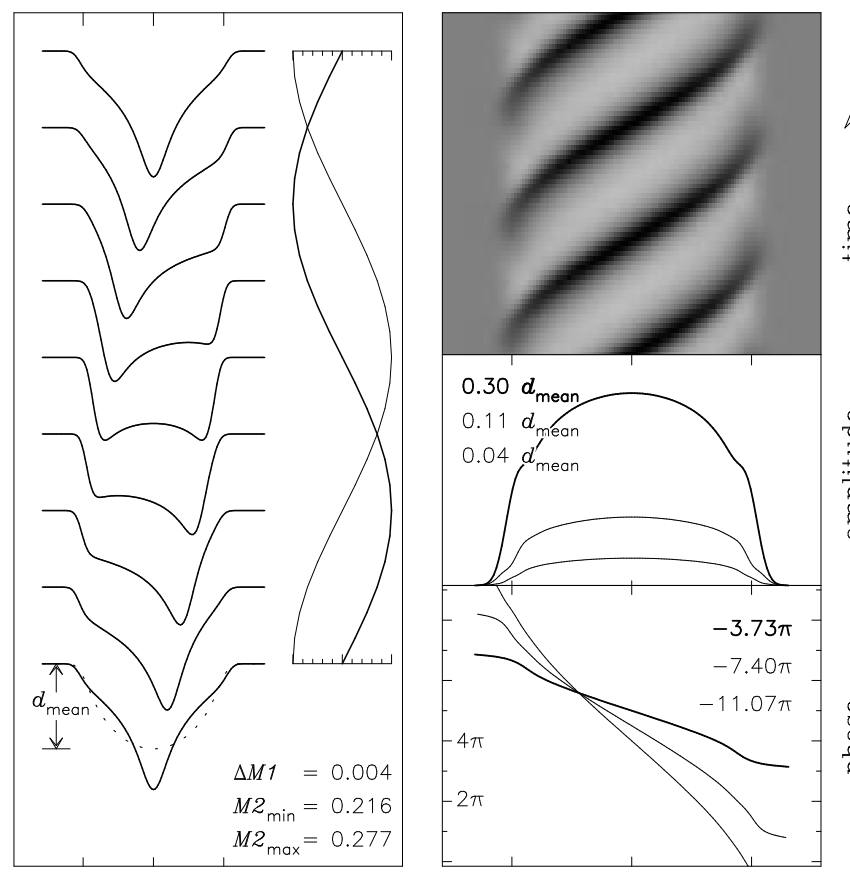

Fig. 2. Example diagram for a pulsation mode with input parameters $\ell=4, m=-4, V_{\max }=0.15 V_{\mathrm{e}} \sin i, k^{(0)}=0.15, W$ $=0.1 V_{\mathrm{e}} \sin i, i=90^{\circ}$, and $\Omega / \omega^{(0)}=0.0$.

Left: Line profiles, and first (thick line, $M 1$ ) and second (thin line, M2) velocity moments during one whole pulsation cycle, with time increasing upwards. The last profile and the first are identical. The tick marks at the velocity axis indicate $V_{\mathrm{e}} \sin i$. The first moment varies sinusoidally around zero with amplitude $\Delta M 1 \times V_{\mathrm{e}} \sin i$. The second moment varies between $M 2_{\min } \times\left(V_{\mathrm{e}} \sin i\right)^{2}$ and $M 2_{\max } \times\left(V_{\mathrm{e}} \sin i\right)^{2}$.

Right: Top: Three pulsation cycles of residual (mean subtracted) spectra are shown in a grey-scale image. Intensities less than average are indicated black; bright regions in the profile are indicated by lighter shades. Middle and bottom: Amplitude distribution and phase diagram. From thick to thin: the amplitude and phase of the line-profile variations at the input frequency $\left(I_{0}(V)\right.$ and $\left.\Psi_{0}(V)\right)$, the first harmonic frequency $\left(I_{1}(V)\right.$ and $\left.\Psi_{1}(V)\right)$, and the second harmonic frequency $\left(I_{2}(V)\right.$ and $\Psi_{2}(V)$ ). In the middle panel, the maximum values of the amplitude distributions are given in units of the average central line depth $d_{\text {mean }}$. The total blue-to-red phase differences $\Delta \Psi_{0}, \Delta \Psi_{1}$, and $\Delta \Psi_{2}$ are given inside the bottom panel, in radians. In all other figures we show only $I_{0}(V), I_{1}(V), \Psi_{0}(V)$, $\Psi_{1}(V)$

the first-harmonic amplitude and phase diagrams, because of the relatively low amplitude of the second harmonic.

For a Gaussian intrinsic profile, the amplitudes of the intensity variations (in continuum units) are proportional to the depth of the intrinsic profile, $d_{\text {intr }}$, and consequently also to the time-averaged depth of the time-series. We give the maxima of the amplitudes across the line profile $\left(I_{0, \max }, I_{1, \max }\right.$, and $\left.I_{2, \max }\right)$ in units of the central depth of the mean of the time series, i.e. we present them in units of $d_{\text {mean }}=P_{\text {mean }}(V=0)$ (see Fig. 2), where $P(V)=1-I(V)$ is the profile function. Therefore, our results are independent of the line depth $d_{\text {intr }}$, and thus applicable to a wide range of absorption lines.

In Fig. 2 we see that the line-profile variations, and therefore the amplitude and phase distributions, extend significantly beyond $V_{\mathrm{e}} \sin i$. In fact, the variability extends to $V_{\mathrm{e}} \sin i+W+$ the maximum projected pulsation velocity, and reading off the phase diagrams at $V_{\mathrm{e}} \sin i$ can lead to a significant underestimate of the blue-to-red phase difference. We measure the blue-to-red phase difference over the full range at which variability is found. The advantage of this is that one does not need to have a priori knowledge of $V_{\mathrm{e}} \sin i$; the disadvantage is that the investigated lines must not be affected by line blending. For blended lines, the phase diagram will continue from one line into the other, and can therefore not be used to determine $\Delta \Psi_{0}$, $\Delta \Psi_{1}, \Delta \Psi_{2}$.

The blue-to-red phase differences $\Delta \Psi_{0}, \Delta \Psi_{1}$, and $\Delta \Psi_{2}$ (printed inside the bottom panel at the right in Fig. 2) are obtained by taking the maximum phase difference between the outermost velocity values at which the corresponding amplitude exceeds $d_{\text {mean }} / 10000$. For real observations, this cut-off velocity will always be smaller because of noise which makes the phase diagram indeterminate at the wings of the profile.

In most cases, the phase diagrams are monotonic over the entire line profile, so that the maximum phase difference $\Delta \Psi$ is found between the outer ends of the region in which variability is found. In some cases, however, there are slope reversals in $\Psi(V)$, so that the maximum difference $\Delta \Psi$ is found in a smaller region in the line profile (see e.g. the middle row of Fig. 7b).

\subsection{The moment variations}

Another important diagnostic for the determination of the properties of line-profile variations is the evaluation of the first few velocity moments of the line profile (Balona 1986; Aerts et al. 1992). It is not our aim to present a detailed study of the moment variations as a function of the different parameters. Such an analysis is presented by De Pauw et al. (1993) in the case of the zero-rotation model. In this work we compute the first and second velocity moment to allow a comparison with De Pauw et al. and Aerts et al. (1992), and to show what global line-profile variations (line shifts, line-width changes) go along with the intensity variations as expressed by the amplitude and phase distributions.

We calculate the first and second velocity moment $M_{1}$ and $M_{2}$, by a weighted summation of the normalized intensity across the line profile

$M_{j} \equiv \int\left(V-V_{\text {ref }}\right)^{j} P(V) \mathrm{d} V$ 
where $P(V)=1-I(V)$ is the profile function and $V_{\text {ref }}$ is a reference velocity. The zeroth moment $M_{0}$ (equivalent width) is constant since local variations of the intrinsic profile are not taken into account here. The first moment is calculated with the rest wavelength of the line as reference, and corresponds to the centroid velocity of the line profile. The second moment is calculated using the first moment as reference velocity $V_{\text {ref }}$, and is a measure of the squared width of the line. We normalize the velocity moments with respect to the equivalent width $M_{0}$, and scale the normalized first and second moments to $V_{\mathrm{e}} \sin i$ and $\left(V_{\mathrm{e}} \sin i\right)^{2}$ respectively. The first velocity moment varies sinusoidally with the pulsation frequency; the second moment shows variations with the pulsation frequency as well as its harmonics.

Our definition of the moments $M_{1}$ and $M_{2}$ is related to the definition of velocity moments $\left\langle v^{j}\right\rangle$ used by Aerts et al. (1992) by

$$
\begin{aligned}
& M_{1}=\frac{<v^{1}>}{V_{\mathrm{e}} \sin i}, \\
& M_{2}=\frac{<v^{2}>}{\left(V_{\mathrm{e}} \sin i\right)^{2}}-M_{1}^{2} .
\end{aligned}
$$

\section{Relevant domains in parameter space}

In the zero-rotation approximation, our model of the lineprofile variations has six parameters. In the slow-rotation model, the relative rotation rate $\Omega / \omega^{(0)}$ is the seventh parameter. Of course, real line profiles and their variations also depend on $V_{\mathrm{e}} \sin i$ and $d_{\text {intr }}$. These two parameters can be removed from the model by proper scalings, which are described in Sects. 4.1 and 5.5. A single representation of the line-profile variability is then applicable to a wide range of rotational velocities and line strengths.

\subsection{Rotation and $\Omega / \omega^{(0)}$}

Following AW, we use values for $\Omega / \omega^{(0)}$ up to 0.5 for the slow-rotation model. The parameters $k$ and $\Omega / \omega^{(0)}$ are closely related because of their joint dependence on $\omega^{(0)}$. Nevertheless, we have chosen to keep them as separate parameters, and consequently, every combination corresponds to a star with different mass, radius and/or rotational frequency. For a given rotational velocity, a large value for $\Omega / \omega^{(0)}$ corresponds to a large $k$-value. This implies that certain domains in the $k^{(0)}-\left(\Omega / \omega^{(0)}\right)$-plane become irrelevant, depending on the ratio, $\eta$, of the centrifugal force to the gravitational force at the equator of the star

$\eta \equiv \frac{\Omega^{2} R_{\mathrm{e}}^{3}}{G M_{*}}=\frac{\left(\Omega / \omega^{(0)}\right)^{2}}{k^{(0)}}$.

In our work we only use combinations of $k^{(0)}$ and $\Omega / \omega^{(0)}$ that satisfy $\eta \leqq 0.25$. This corresponds to an equatorial rotation velocity that is at most $50 \%$ of the equatorial break-up velocity. Clement (1994) has found that the spheroidal part of axisymmetric modes (zonal, $m$ $=0$ ), does not maintain its basic zero-rotation spatial distribution. Therefore, our model might be inaccurate for zonal modes with high $\Omega / \omega^{(0)}$.

\subsection{Prograde and retrograde modes}

The frequencies that we use for our line-profile calculations are those as observed in the corotating frame of the star. In this way the retrograde modes lead to bumps that move from red to blue in the line profiles, while the bumps of prograde modes move from blue to red. This makes it easier to distinguish prograde and retrograde modes in our figures. In the zero-rotation approach, the line-profile variations associated with prograde $(m<0)$ and retrograde $(m>0)$ modes behave symmetrically in time (see Fig. 6, right two columns). This symmetry is broken by the effects of rotation on the mode, which necessitates a separate investigation of prograde and retrograde modes in the case of the slow-rotation model.

\subsection{The inclination angle $i$}

Symmetries in the expressions of the displacement field allow us to restrict the inclination angle $i$ to the interval $\left[0^{\circ}, 90^{\circ}\right]$ (Aerts 1993).

\subsection{The use of $V_{\max }$ instead of $a_{\mathrm{sph}}$}

A comparative study of line profiles with different values of $\ell, m, k^{(0)}$, or $\Omega / \omega^{(0)}$, in which the model amplitude $a_{\text {sph }}$ is kept constant, is not convenient, since this implies physical situations with substantially different velocities, and correspondingly different amplitudes of the line-profile variations. To facilitate a fair comparison between the line-profile variations resulting from different parameter settings, we adjust the displacement amplitude $a_{\text {sph }}$ in each case such that the calculated maximum pulsational surface velocity

$$
V_{\max } \equiv\left(\sqrt{V_{\mathrm{r}}^{2}+V_{\theta}^{2}+V_{\phi}^{2}}\right)_{\max }
$$

is kept the same whereas the other parameters may vary.

\subsection{The use of $V_{\mathrm{e}} \sin i$ as a scaling factor}

Two profiles will have identical shapes if the combination of $V_{\max } / V_{\mathrm{e}} \sin i$ and $W / V_{\mathrm{e}} \sin i$ is the same for both profiles. Such profiles differ only in velocity (wavelength) and intensity scale. We therefore use $V_{\mathrm{e}} \sin i$ as a scaling factor for the velocity amplitude, for the width of the intrinsic profile, and for the velocity scale of the line profile. We adopt only one specific value of $V_{\mathrm{e}} \sin i$ and vary the pulsation amplitude and intrinsic width to investigate the response in the line-profile variations.

The amplitude of pulsation $V_{\max }$ and the intrinsic profile width $W$ have a broad range of physically relevant 
values. The more rapid rotators are best accounted for by relatively small values of $V_{\max } / V_{\mathrm{e}} \sin i$ and $W / V_{\mathrm{e}} \sin i$, while the slower rotators have values of $V_{\max }$ and $W$ of order $V_{\mathrm{e}} \sin i$.

\subsection{The ratio $k^{(0)}$ of the horizontal to the vertical amplitudes}

Theoretically, the value for $k$ can range from approximately zero up to infinity (Eq. 22). If we keep $V_{\max }$ the same for each calculated mode, the profiles do not change much for $k$ values higher than a certain value $k_{+}$. Above this value, the magnitude of the velocity vector is almost proportional to $k$, because the radial velocity component becomes negligible. For all investigated modes we found $k_{+} \gtrsim 1$, depending somewhat on the considered $\ell$ value. In Figs. 7 and 9, where $k^{(0)}$ is one of the two running parameters, we adjust the range of $k^{(0)}$ according to the value of $k_{+}$. The line-profile characteristics for the highest values that we use for $k^{(0)}$ can be considered as representative for all higher values as well.

For the calculation of line profiles, we have chosen to use $k^{(0)}$ as model parameter and to calculate the corrected $k$ from Eq. (22). Such an approach allows to separate the effect of the rotation on $k$, and to study the line profiles by comparing different rotation rates for the same $k^{(0)}$-value (Fig. 9).

\section{Results}

We computed a large number of time series of line profiles on two-dimensional grids. For each grid we varied 2 out of 7 relevant pulsational and stellar parameters: $\ell, m$, $V_{\max }, k^{(0)}, i, \Omega / \omega^{(0)}$, and $W$. From these calculations, we selected a few grids to serve as illustrations in this paper (Figs. 3-9). The domains of the parameters in these grids are chosen to bring out the most illustrative examples, while covering the physically relevant cases. The results of each grid calculation are displayed in two parts. The left part displays the time series of line profiles along with the corresponding behavior of the first and the second velocity moment. The right part shows the corresponding residual spectra, and the distributions of variational amplitudes and phases across the line profile. See Fig. 2 for an example.

Note that in Figs. 3-9 we present time series of line profiles during 3 pulsation cycles, without specifying the time scale of the variability; the velocity axes are scaled to $V_{\mathrm{e}} \sin i$. This means that in some of the Figs. 3-9, the parameters that change along the axes of the grid imply stars with different mass, radius, rotation period and/or pulsation frequency.

\subsection{Line-profile variations with the pulsation frequency and its harmonics}

As shown in Sect. 4.1, line-profile variability is in general not only found at the input frequency of the variations but also at its multiples (i.e. its harmonics), even for an assumed sinusoidal behavior of the surface velocity field (e.g. for $k=0$ and $\Omega / \omega^{(0)}=0$ ) (see also Fig. 1). This effect is due to the Doppler-mapping of the three-dimensional stellar surface velocity field onto one-dimensional velocity (or wavelength) space, which has only a one-to-one correspondence $^{1}$ in the limit of zero pulsation amplitude. Sharply peaked moving bumps give rise to strongly nonsinusoidal line-profile time-variability, which results in large harmonic amplitudes.

6.1.1. The amplitude distribution at harmonic frequencies

The shape of the amplitude distribution across the line profile depends on all parameters, in many cases in a very complicated manner. Only the behavior of the amplitude distribution of sectoral modes with different values of $k$ are easy to understand (e.g. see Sects. 6.2 and 6.3). In general, the shape of the amplitude distribution of the variations at the harmonic frequency resembles that at the input frequency.

The relative magnitude of the variations at the different frequencies is another aspect of the variability. The ratio of the variational amplitude at the first harmonic frequency to that at the input frequency, $I_{1}(V) / I_{0}(V)$, is an indicator of the non-sinusoidal nature of the line-profile variations, and depends on the position in the line profile. We write the ratio of the maxima of the individual distributions (that may occur at different velocity positions) as $\left(I_{1} / I_{0}\right)_{\max }$. We find that for all investigated cases $\left(I_{0} / I_{2}\right)_{\max }>1$ and $\left(I_{1} / I_{2}\right)_{\max }>1$. For most but not all cases we find $\left(I_{0} / I_{1}\right)_{\max }>1$.

We find that $\left(I_{1} / I_{0}\right)_{\max }$ depends mostly on $W$ and on $V_{\max }$. This is illustrated in Fig. 8. The relative harmonic amplitudes $\left(I_{1} / I_{0}\right)_{\max }$ and $\left(I_{2} / I_{0}\right)_{\max }$ increase for increasing pulsation amplitude $V_{\max }$. The width of the intrinsic line profile causes a blurring of the moving bumps. Therefore, the contrast (sharpness) of the bumps will decrease with increasing intrinsic width, and consequently also $\left(I_{1} / I_{0}\right)_{\max }$.

We find weaker dependences of $\left(I_{1} / I_{0}\right)_{\max }$ and $\left(I_{2} / I_{0}\right)_{\max }$ on $k$ and $i$. A small decrease of $\left(I_{1} / I_{0}\right)_{\max }$ is found towards the high $k$-values and low inclination angles.

\footnotetext{
1 For a non-pulsating uniformly rotating spherical star there is a one-to-one relation between a straight line on the stellar disk, parallel to the stellar rotation axis, and a position in the line profile: the integrated stellar light emitted by the surface elements on this line is Doppler-mapped to a single position in the line profile by the rotation of the star.
} 
In Fig. 5 we illustrate the peculiar line-profile behavior that we found for modes with $\ell-m$ an odd number and with an inclination angle very close to $90^{\circ}$. For these modes, we find that the apparent number of moving bumps in the line profile is doubled. This behavior leads to a variability that is found at even multiples of the input frequency, but not at the input frequency itself (Fig. 5, see Reid \& Aerts 1993). Towards an inclination of $90^{\circ}$ the variation at the input frequency disappears because of cancellation effects, whereas the variability at the first harmonic is unaffected. The cancellation at the input frequency is caused by the symmetric behavior of the pulsation with respect to the stellar equator, and will already disappear at inclinations that differ only a few degrees from $90^{\circ}$ (see Paper III).

6.1.2. Determination of $\ell$ and $|m|$ from the phase differences across the line profile

If the power at the harmonics relative to that at the input frequency is small, the blue-to-red phase difference $\Delta \Psi_{0}$ is directly related to the number of traveling bumps that migrate through the line profile. In such cases of sinusoidal line-profile variations, the phase difference across the line profile can be measured by counting the number of traveling bumps. We find that the phase diagrams themselves provide a more objective measure of the number of cycles across the line profile, regardless of the importance of the harmonics. For multiple modes, the number of traveling bumps in the line profile is determined by the combined effect of all modes, in which case the blue-to-red phase differences can only be successfully determined if the apparent frequencies of each of the modes can be resolved (see Paper II).

Many authors (e.g. Smith 1986; Gies \& Kullavanijaya 1988; Kambe \& Osaki 1988; Yang et al. 1988; Kambe et al. 1990) have used the number of visible bumps or, equivalently, the blue-to-red phase difference $\Delta \Psi_{0}$ to identify $|m|$ according to $\Delta \Psi_{0}=|m| \pi$. However, from our calculations we find that the line-profile behavior for both tesseral and sectoral modes displays a blue-to-red phase difference proportional to $\ell$ rather than to $|m|$. Such a relation was already suggested by Merryfield \& Kennelly (1993), who found for one tesseral mode that their apparent azimuthal order $\hat{m}$ corresponds more closely to $\ell$ than to $|m|$. In addition, we find that the phase difference $\Delta \Psi_{1}$ at the first harmonic frequency $2 \omega_{\text {obs }}$ is an indicator of $|m|$. Figure 4 gives a typical example of this behavior of the phase diagrams.

We tentatively conclude that it is possible to determine both the degree $\ell$ and the azimuthal order $|m|$ from the phase information provided by a Fourier analysis of an observed time series of high-resolution spectra with sufficient signal to noise ratio; in Paper II we elaborate on this subject. An independent check of the $(\ell,|m|)$-value can be performed by an application of the moment method (for $l \lesssim 4$, and if effects of rotation and temperature variation can be neglected).

In several of our diagnostic diagrams (see Figs. 6 and 9) for modes with intermediate to high $k$-values, we find a blue-to-red phase difference $\Delta \Psi_{0}$ that differs by $2 \pi$ from the empirical relation with $\ell$ that is shown by the majority of our calculations. Modes with low $k$-values show line-profile variations mainly caused by radial motions, causing a phase of variability that changes across the line profile along with the change of phase of the traveling waves at the surface of the star. For intermediate to high $k$-values the profile variations do not follow the continuous phase change at the stellar surface. A positive azimuthal motion $\left(V_{\phi}>0\right)$ will cause a negative Doppler-shift at the blue wing of the profile, but a positive shift at the red wing. This results in an additional change of phase by $\pi$ around the line center. Other sources of variability at the line center determine whether this change will be positive or negative, which leads to differences of $2 \pi$ in $\Delta \Psi_{0}$ for modes with the same $\ell$-value. The blue-to-red phase difference of modes with intermediate to high $k$ is therefore co-determined by other possible sources of variability at the line center (e.g. toroidal terms; radial and $\theta$-movements; local brightness and EW changes). In this work, differences in $\Delta \Psi_{0}$ of $2 \pi$ are found by using different parameters $i$ and $\Omega / \omega^{(0)}$ (see Figs. 6 and 9 ).

A detailed analysis of the determination of $\ell$ and $|m|$ from the phase diagrams at the input frequency and its harmonics is the subject of investigation in Paper II.

\subsection{The effects of rotation on the line-profile behavior}

The effects of the Coriolis force on the line-profile variations are hard to classify, because they depend on the specific combination of parameter values. However, the effects of the Coriolis force increase for higher values of $k$.

For high-degree sectoral modes (say $\ell>4$ ), the effects of rotation are negligible for values of $\Omega / \omega^{(0)}$ up to 0.5 . This is because the toroidal amplitude of these sectoral modes is too small, compared to the spheroidal amplitude, to have a large effect on the line profiles. Under the same conditions the value of $k$ is also not modified by rotation (Eq. 22). The effects of rotation increase in importance for sectoral modes of lower degree. If $\ell$ is small enough $(0,1$ or 2) the first-order terms can lead to drastic changes in the line profiles and their variations.

Figure 3 shows the line-profile behavior of a prograde sectoral $\ell=2$ mode and a radial $(\ell=0)$ mode. It shows that radial modes in rotating stars can easily be confused with $\ell=-m=2$ modes. In spite of the completely different surface velocity fields of these modes, the presence of rotation induces a very similar line-profile behavior. The reason for the similarity is purely accidental; in case of the $\ell=2$ mode the dominant rotational effect is a change in $k$-value, whereas the radial mode is changed only by one toroidal term (see Eq.12) which induces a periodic 
$\left(\Omega / \omega^{0}\right)=0.010$
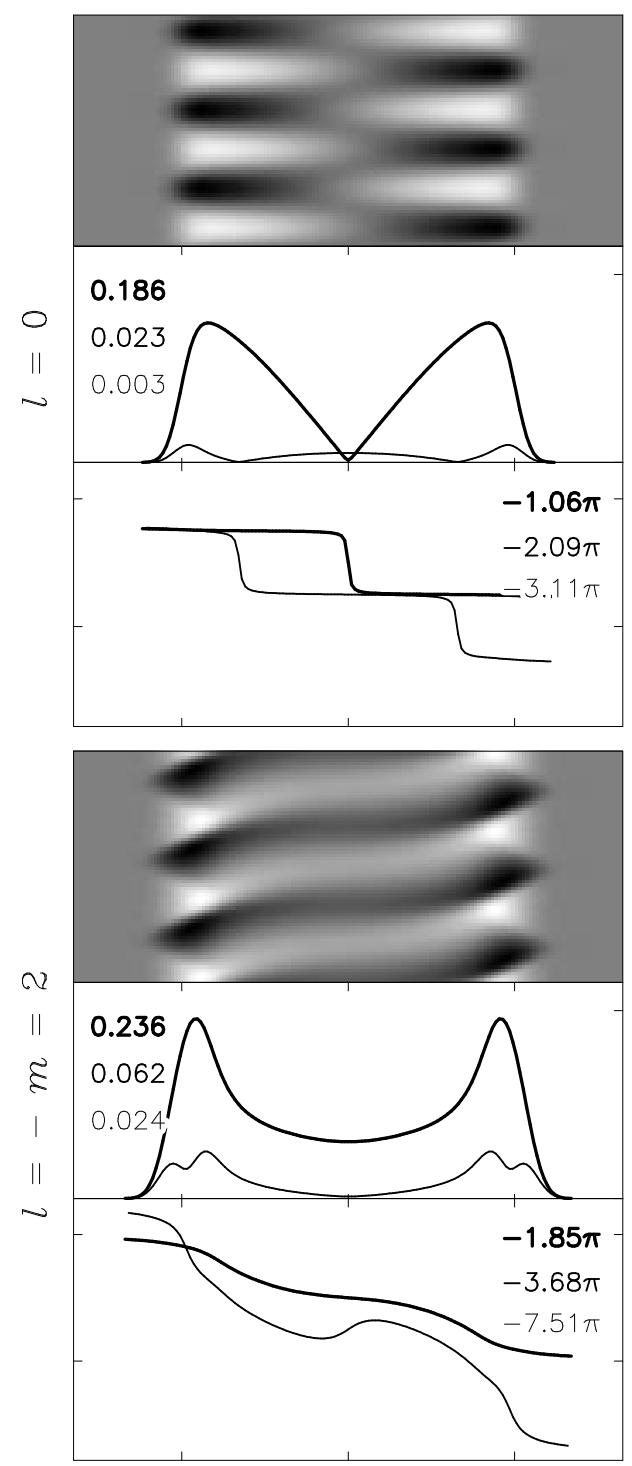

$\left(\Omega / \omega^{0}\right)=0.200$
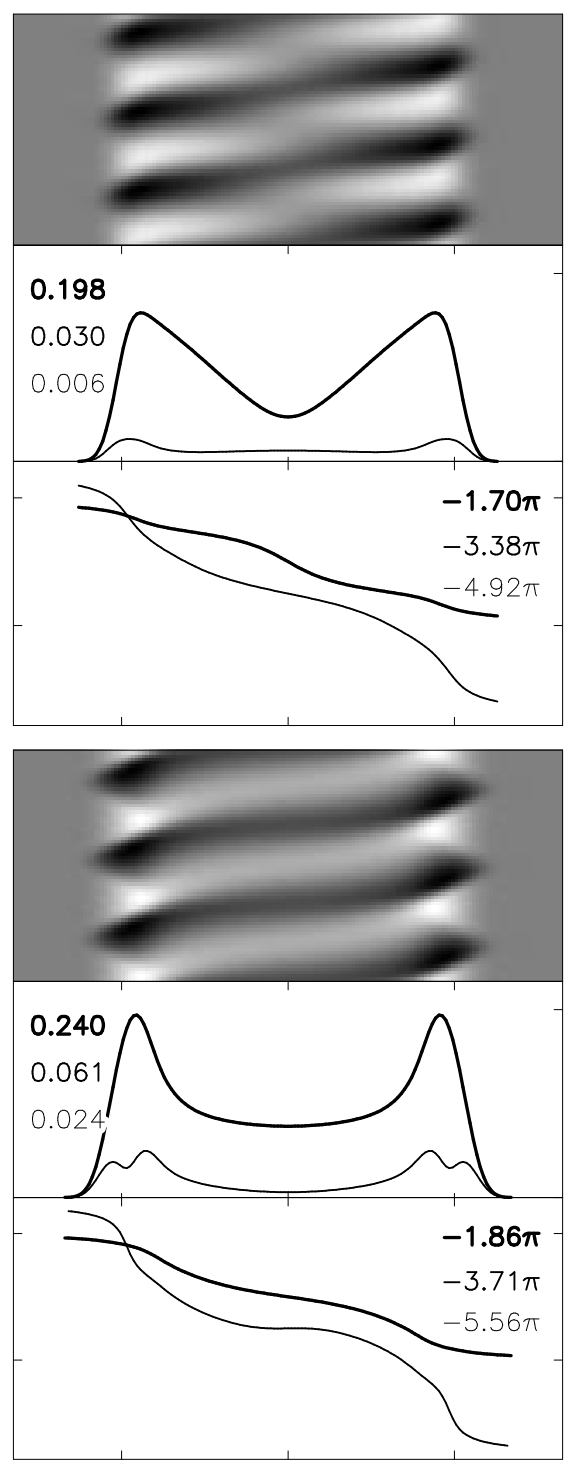

$\left(\Omega / \omega^{0}\right)=0.400$
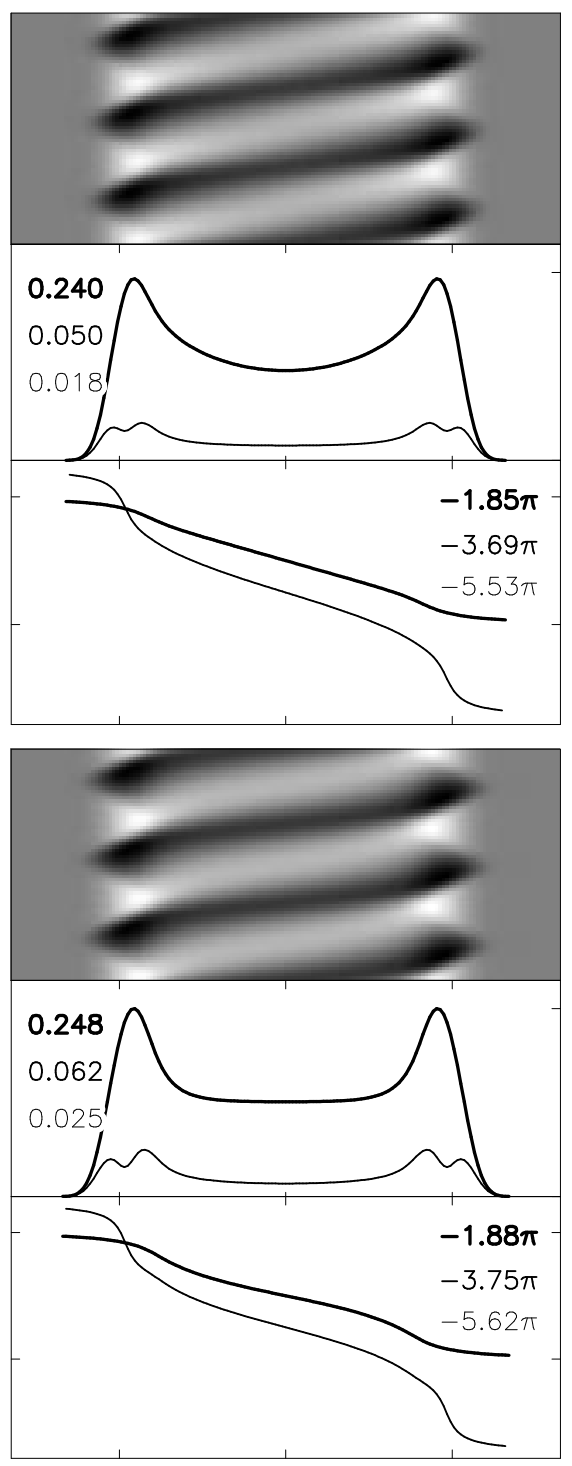

Fig. 3. Line-profile variability for two different pulsation modes as a function of $\Omega / \omega^{(0)}$. See Fig. 2 for a description of the figures. For clarity we do not plot $I_{2}(V)$ and $\Psi_{2}(V)$. Top row: radial pulsation $(\ell=0)$. Bottom row: non-radial pulsation mode $(\ell=2, m=-2)$. The values of the other parameters are (for both modes): $V_{\max }=0.15 V_{\mathrm{e}} \sin i, W=0.1 V_{\mathrm{e}} \sin i, k^{(0)}=0.8($ not relevant if $\ell=0$ ), and $i=90^{\circ}$. The effect of the rotation is large for these low-degree modes. In both cases power is added to the line center, leading to similar line-profile behavior. For the radial mode this is caused by the rotationally induced toroidal term; for the non-radial mode the effect is mainly caused by the first-order correction of $k$

increase/decrease of the rotational velocity at the surface. Note the similarity with the line-profile variations presented by Gies (1994).

Tesseral modes are much more affected by the Coriolis force than sectoral modes since for tesseral modes the rotation gives rise to a relatively large second toroidal correction term (see Eq. 12), which is zero for sectoral modes. We find remarkable line-profile behavior for tesseral modes with a high $k^{(0)}$-value and no node line at the equator $(\ell$ - $m$ even). In the slow-rotation model, these tesseral modes lead to dominant variability at the line center, similar to a sectoral mode with small $k^{(0)}$-value (see Figs. 6).

In his discussion of the so called $k$-problem, Smith (1986) mentioned that for high $k^{(0)}$-values, the toroidal term(s) caused by rotation might be able to mimic the amplitude-distribution characteristics of a low- $k^{(0)}$ mode. For sectoral modes with $\ell>3$ and values of $\Omega / \omega^{(0)} \leqq 0.5$, we find that the toroidal term induced by the Coriolis force is not capable of generating enough variability near the line center. However, as mentioned above, tesseral modes are modified by a second toroidal term that can indeed 
contribute to the amplitude at the line center, mimicking the characteristics of a low- $k^{(0)}$ mode.

For the line-profile variations that are significantly affected by the effects of the Coriolis force, we find differences between the prograde and the retrograde modes. We find only modest differences between the amplitude diagrams of both cases. As mentioned in Sect. 6.1.2, the blue-to-red phase difference of modes with intermediate to high $k$ values is co-determined by the toroidal motions caused by the Coriolis force. In some cases this leads to a difference of $\approx 2 \pi$ between the prograde and the retrograde case (see rightmost 2 columns in Fig. 6).

\subsection{The difficulty to derive $k$-values from amplitude diagrams}

The determination of the $k$-value from observed lineprofiles has been discussed by several authors (e.g. Smith 1986; Kambe et al. 1990; Lee \& Saio 1990). Many factors may play a role in the appearance of modes with high $k$-values, of which a few are discussed below.

For sectoral modes, the most significant effects of the parameter $k$ on the line-profile behavior can be described as follows (see Fig. 7, top row). A convex amplitude distribution is found for $k \sim 0$. For a somewhat higher value of $k$ (depending on the values of $m$ and $\ell$ ), the amplitude distribution takes a rectangular shape, as a consequence of larger horizontal motions. This trend persists towards the higher $k$-values so that above a certain value of $k$ all variability is concentrated in the wings of the absorption profile, and the amplitude distribution has acquired a double-peaked shape with almost no power in the line center. Proceeding to still higher values of $k$, while keeping $V_{\max }$ constant, has no further effect on the line profiles and their amplitude distribution, since the horizontal motions already dominate.

The rectangularly shaped and double-peaked amplitude distributions of sectoral modes with intermediate to high $k$-values are blurred at high values of the intrinsic width $W / V_{\mathrm{e}} \sin i$ (see Fig. 7). If $W / V_{\mathrm{e}} \sin i$ is sufficiently large, a change in $k$ does not lead to any effect on the amplitude distribution at all. A correct determination of $k$, by means of amplitude distributions, should therefore include a reasonable estimate for the intrinsic line-profile width $W / V_{\mathrm{e}} \sin i$. This is especially relevant for line profiles of light ions in slow/moderate rotators.

The $k$-characteristics of tesseral modes depend crucially on the relative rotation rate $\Omega / \omega^{(0)}$. As we have shown in Sect. 6.2 and Fig. 6, slow rotation changes the amplitude distribution of many high- $k$ tesseral modes in such a way that the highest amplitude is found at the line center, whereas for other high- $k$ tesseral modes the maximum amplitudes are found in the wings of the profile. Therefore, we cannot identify high- $k$ characteristics for tesseral modes in general.
Finally, it has been shown previously (Lee \& Saio 1990; Lee et al. 1992; Townsend 1996) that if temperature effects (i.e. local surface brightness and EW variations) codetermine the line-profile formation, the $k$-characteristics may generally disappear. We intend to describe the influence of these temperature effects on the amplitude and phase diagrams in a separate paper (Paper IV).

We conclude that in general it is difficult to determine the $k$-value of a pulsation mode from the amplitude distributions of observed time series of line profiles alone. However, a double-peaked amplitude distribution always reflects a high $k$-value, if the blue-to-red phase difference $\Delta \Psi_{0}$ exceeds $2 \pi$ radians. A double-peaked amplitude distribution together with a blue-to-red phase difference of less than $2 \pi$, can also occur for a radial or an $\ell=1$ mode.

\subsection{Amplitudes of line-profile variations of tesseral modes}

It is well known that, if the star is seen equator-on, sectoral modes are much more effective in producing line-profile variations than tesseral modes. For smaller inclination angles, this effectiveness is reduced. For tesseral modes, this efficiency behaves different with respect to the inclination angle. Towards smaller inclination angles, tesseral modes are increasingly efficient in producing line-profile variability. In general one could state that at inclinations around $45^{\circ}$ the tesseral modes are more efficient in producing lineprofile variations than sectoral modes, for equal surface velocities. This aspect of tesseral modes is illustrated in Figs. 5.

As expected, the line-profile amplitude characteristics of tesseral modes are much more sensitive to the inclination angle, than those of sectoral modes. Especially in the cases that the Coriolis force causes significant toroidal terms, the line-profile formation of tesseral modes is very complex (see Sect. 6.2).

\subsection{Retrieval of the inclination}

We have not found a clear indication that one can derive the inclination from the IPS diagnostics. The presence of red-to-blue moving bumps, due to the traveling waves at the far side of the star, coexisting with blue-to-red moving bumps might be indicative for a low $i$ (see Baade 1984, 1987). The presence of coexisting bumps moving in opposite directions, has been discussed by Kambe \& Osaki (1988) for the case of toroidal modes with small inclinations. Since for some modes there is enough information in the line-profile variability to derive $i$ with the moment method, we conclude that it should be possible to estimate the inclination by fitting generated time-series of line profiles or IPS amplitude and phase diagrams to the observed ones. However, such an attempt can only be successful if both $\ell$ and $m$ are precisely known. 


\section{Conclusions}

We presented a description of the oscillatory displacement field at the surface of a non-radially pulsating rotating star. This description accounts for the effects of the Coriolis force on the pulsation, and is relatively simple to implement in models.

We modeled time series of line profiles of rotating stars that exhibit non-radial pulsations, and obtained amplitude and phase diagrams by means of a Fourier decomposition of the intensity variations in the line profile. We found a number of interesting aspects of the line profiles and their temporal behavior, which are either new or have not been mentioned elsewhere.

The precise shape of the intrinsic profile function only affects the characteristics of the line-profile variability if the width of the intrinsic line profile becomes of the order of the projected equatorial rotation velocity $V_{\mathrm{e}} \sin i$.

Limb darkening does not fundamentally change the line-profile behavior. The phase diagrams are hardly affected by limb darkening. However, the amplitudes of intensity variations in the normalized line profiles do depend somewhat on the limb-darkening coefficient.

For a sufficiently inclined star, tesseral modes can produce large line-profile variations; the variations can become even larger than those of a sectoral mode.

For large pulsational velocities, harmonics of the observed pulsation frequency are needed to describe the lineprofile variability. The larger the line-profile variations caused by the velocity variations, the larger is the contribution of harmonics to the variations. The harmonic variability increases also for a decreased intrinsic line profile width. We found a steep increase of the relative harmonic contribution, i.e. an apparent doubling of the number of bumps in the line profile, for modes with odd $\ell-m$ with an inclination close to $90^{\circ}$.

The apparent number of bumps and troughs in the line profile is a measure of $\ell$ only if the harmonics of the line profile variations are relatively unimportant, and if the star is pulsating in a single mode. However, one can circumvent these restrictions with a Fourier analysis of the time behavior of line profiles: the degree $\ell$ and the azimuthal order $m$ are related to the blue-to-red phase differences at the apparent frequency and its first harmonic respectively. It should therefore be possible to derive $\ell$ and to put constraints on $|m|$ from IPS phase diagrams.

The ability to detect high- $k$ characteristics in the lineprofile variations depends on the width of the intrinsic line profile, $W$ : detection is only possible for cases with $W \lesssim 0.4 V_{\mathrm{e}} \sin i$. For tesseral modes, we find no characteristics that distinguish between low and high $k$-values; a large subset of the tesseral modes with a high value of $k$ still gives variability in the line center. In the general case, without presuming that the observed line-profile variations are due to a sectoral mode, we find no characteristics of the variations that make it possible to derive a conclusive value of $k$. Only the cases with a double-peaked amplitude distribution give unambiguous information by explicitly implying a high $k$-value, provided that the corresponding phase difference $\Delta \Psi_{0}$ exceeds $2 \pi$.

The effects of slow rotation on the line-profile behavior of sectoral modes, are only important for modes of lowdegree. Line-profile variations of tesseral modes can be heavily affected by the effects of rotation. The rotationally induced toroidal movements of tesseral modes with even $\ell-|m|$ and with large $k$-values give rise to a line-profile behavior which is similar to that of sectoral modes with low $k$-values. The line-profile variability of a radial mode in a rotating star can easily be confused with that of a lowdegree non-radial mode, if the Coriolis force is important.

Our study of line-profile behavior has led to an atlas containing line profiles and their characteristics, for various values of the pulsation and relevant stellar parameters. This atlas can serve as a useful guide for those who plan to perform an analysis of observed line-profile variations in many types of rotating pulsating stars. 


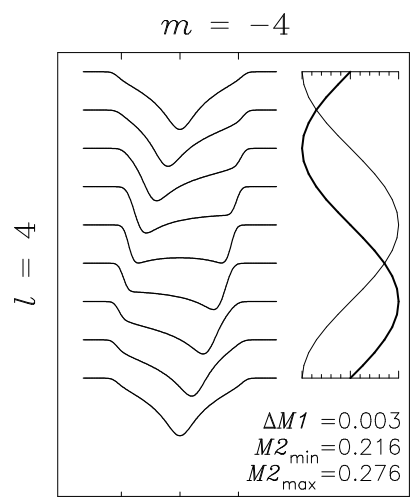

$m=-6$

$m=-8$

$m=-10$
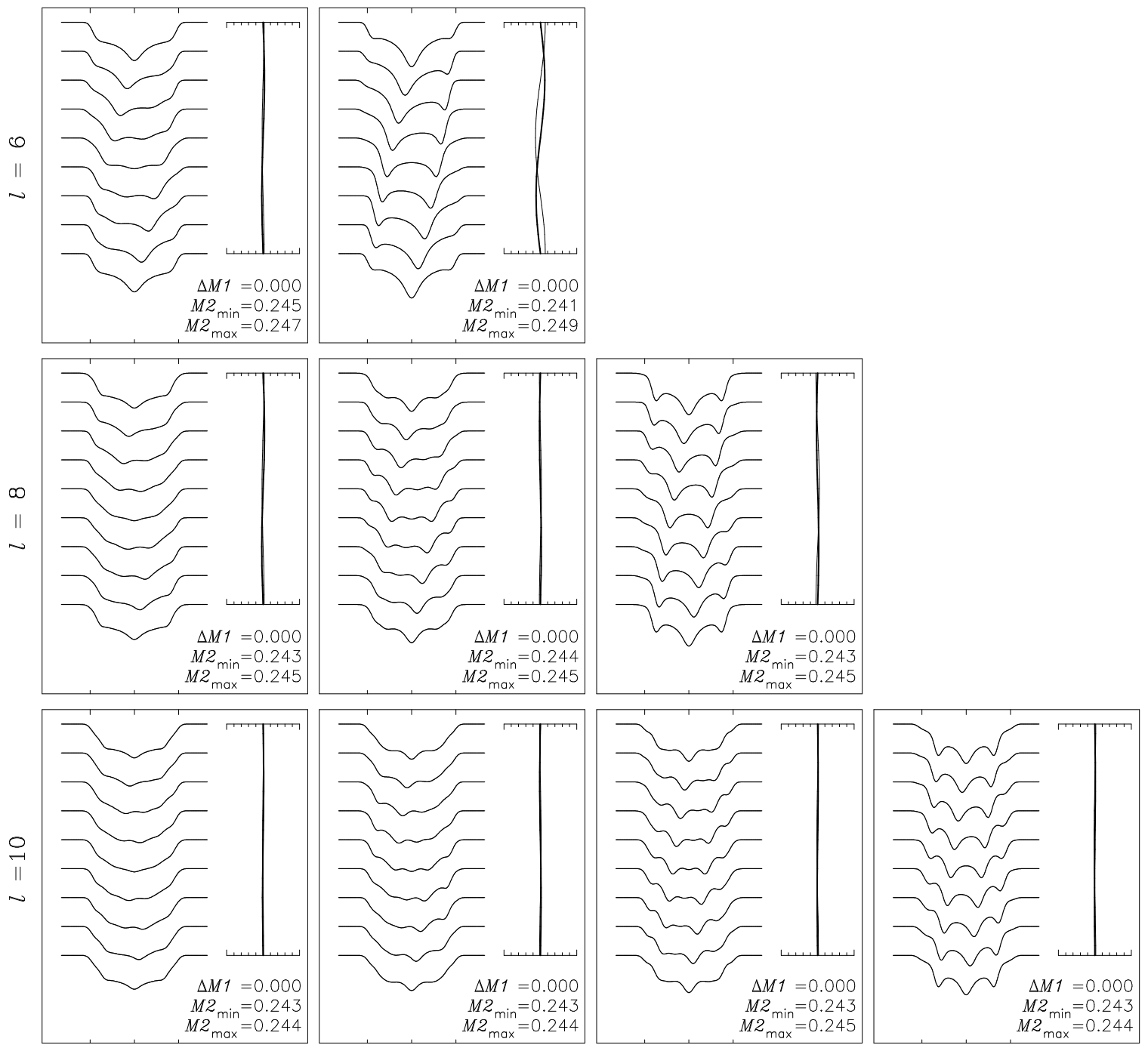

Fig. 4. a) Time series of line profiles and their first and second velocity moments, calculated for a grid of different combinations of $\ell$ and $m$. The remaining fixed parameters are: $i=70^{\circ}, \Omega / \omega^{(0)}=0.0, W=0.10 V_{\mathrm{e}} \sin i, V_{\max }=0.15 V_{\mathrm{e}} \sin i, k^{(0)}=0.20$. The first velocity moments are all plotted at the same scale, as well as the variations of the second moment. The scales at which the moments are plotted are therefore determined by the maximum moment variations that are found in the grid. See Fig. 2 for a detailed description of the figures. Here we show that the variations of the velocity moments are negligible for high-degree modes $(\ell \gtrsim 4)$. Variations of the first two velocity moments of high-degree modes (say $\ell>4$ ) will be very hard to detect with present observational techniques. Due to the nearly edge-on perspective, the line-profile variations of the tesseral modes are systematically low; they increase for smaller inclination angles (see Figs. 5) 


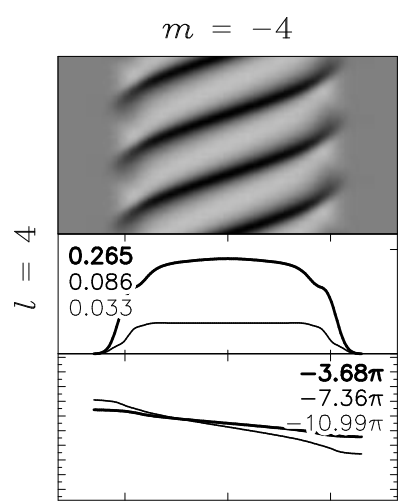

$m=-6$

$m=-8$

$m=-10$
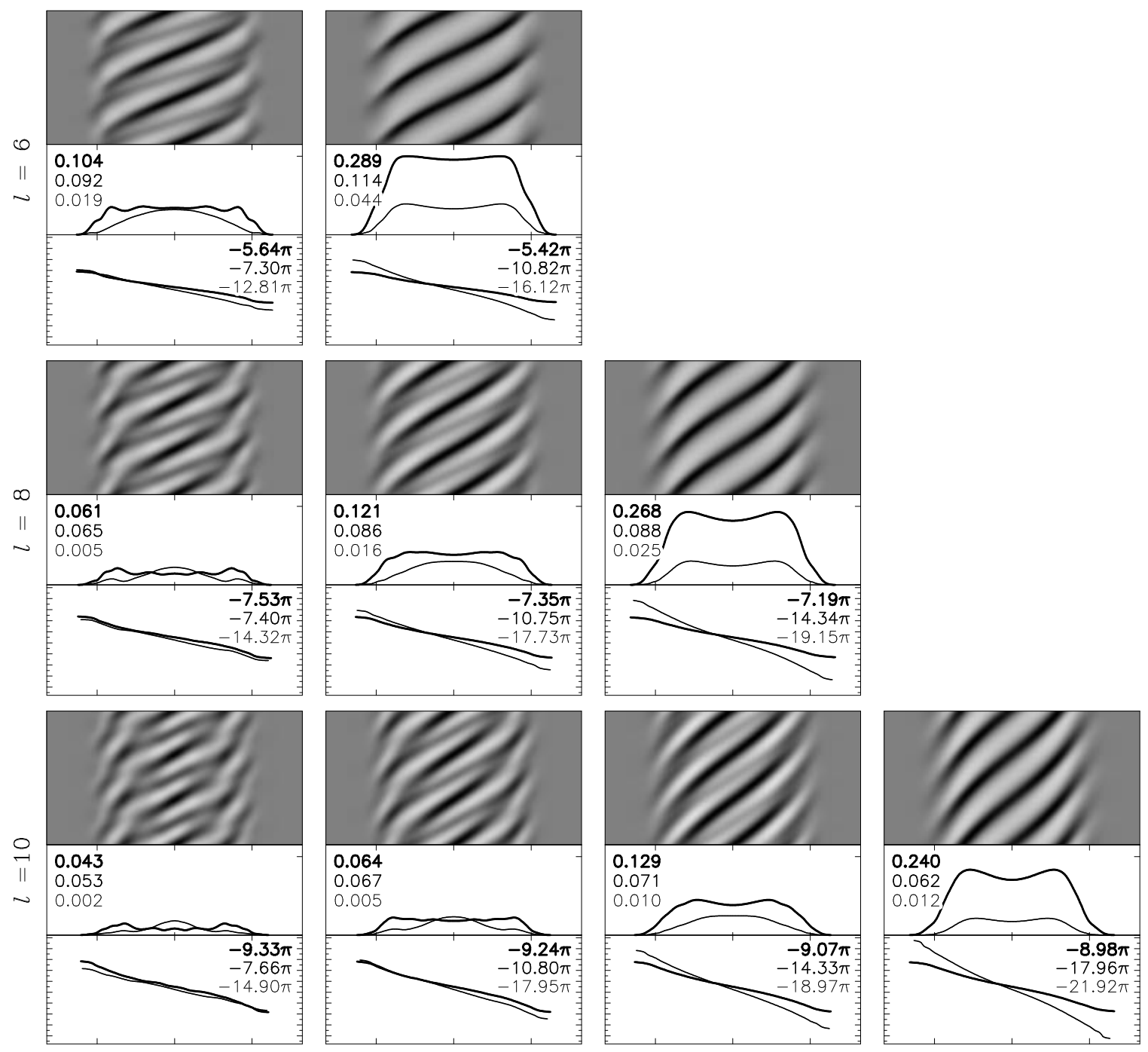

Fig. 4. b) Residual spectra, amplitude distributions $I_{0}(V)$ and $I_{1}(V)$, and phase distributions $\Psi_{0}(V)$ and $\Psi_{1}(V)$ of the time series of line profiles displayed in Fig. 4a. For a detailed description of the figures, see Fig. 2. Note that in the gray-scale images we show three pulsation cycles of the line-profiles without specifying the time-scale of the variability. In the panels with amplitude and phase diagrams we give the maxima of the amplitude distributions $I_{0}(V), I_{1}(V), I_{2}(V)$ in units of $d_{\text {mean }}$, and the total blue-to-red phase differences $\Delta \Psi_{0}, \Delta \Psi_{1}$, and $\Delta \Psi_{2}$ in radians. We only show amplitude and phase diagrams for the input frequency $\left(I_{0}(V), \Psi_{0}(V)\right)$ and its first harmonic $\left(I_{1}(V), \Psi_{1}(V)\right)$. The grid on this page gives a typical example of the blue-to-red phase difference of the variations at the input frequency, $\Delta \Psi_{0}$, being an indicator of $\ell$. This finding rejects earlier suggestions that the number of bumps in the line profiles is proportional to $|m|$. The blue-to-red harmonic phase difference of the variations, $\Delta \Psi_{1}$, does show to be an indicator of $|m|$ 

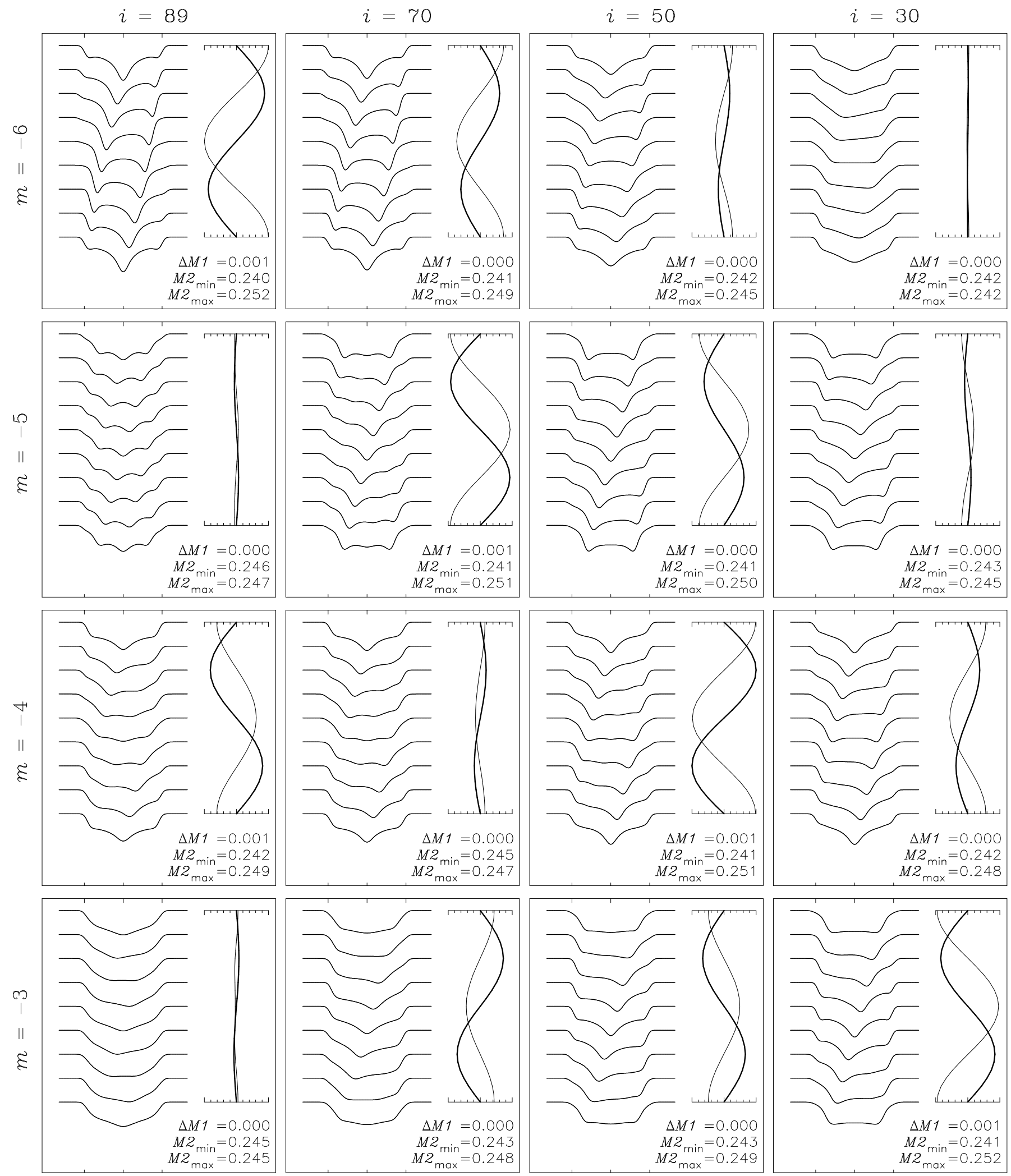

Fig. 5. a) Same as Fig. 4a but for inclination $i$ and azimuthal order $m$. Fixed parameters are: $\ell=6, \Omega / \omega^{(0)}=0.0, W=0.10$ $V_{\mathrm{e}} \sin i, V_{\max }=0.15 V_{\mathrm{e}} \sin i, k^{(0)}=0.20$. Note that the velocity moments, which are very sensitive to the inclination angle, have a far more detailed dependence on $i$ than what can be seen from this $4 \times 4$ grid 

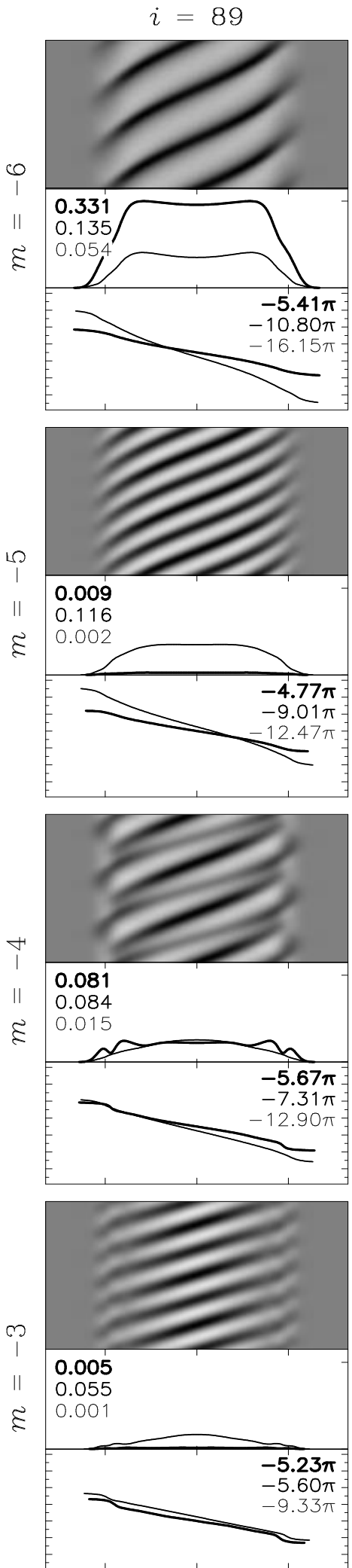

$i=70$

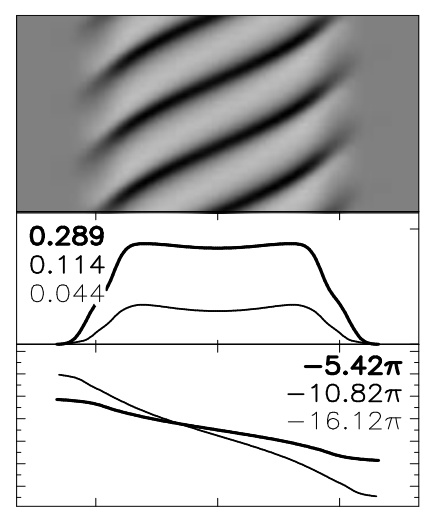

$i=50$
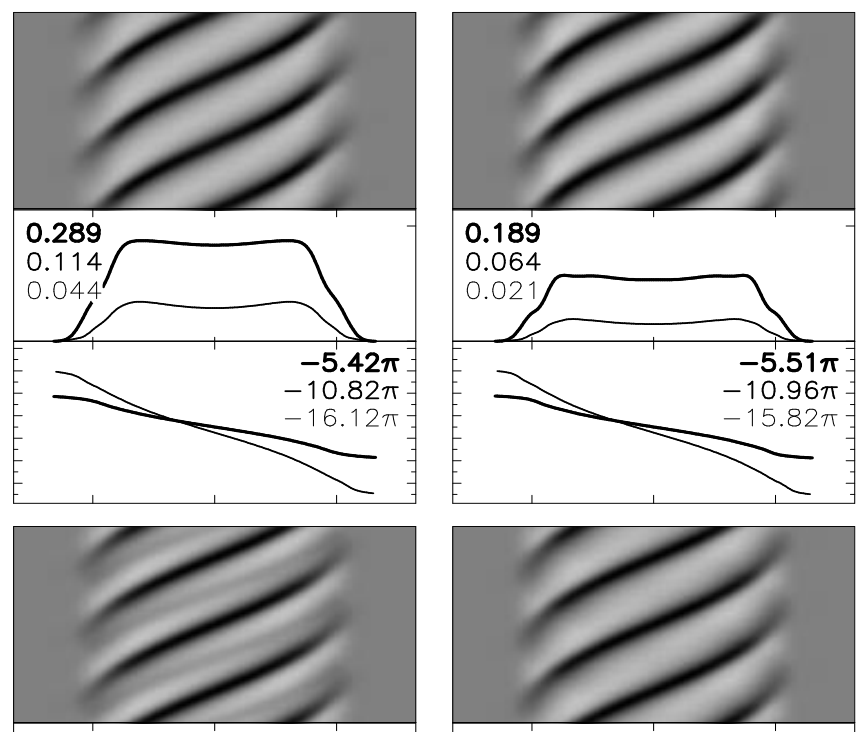

\subsection{2}

0.110
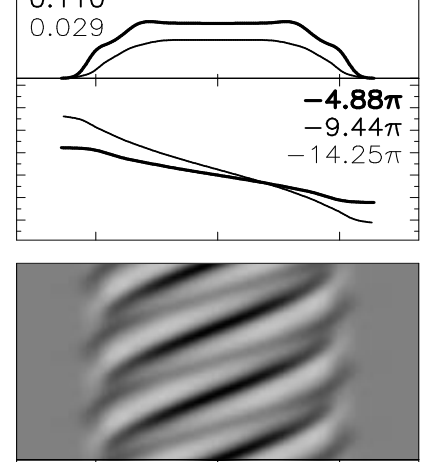

0.104

0.092
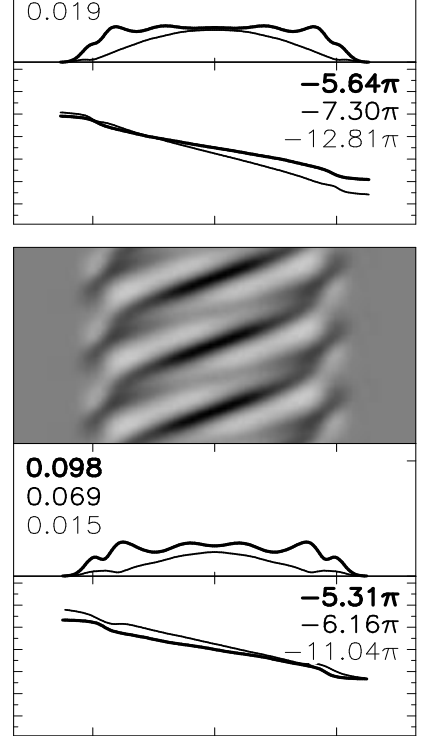
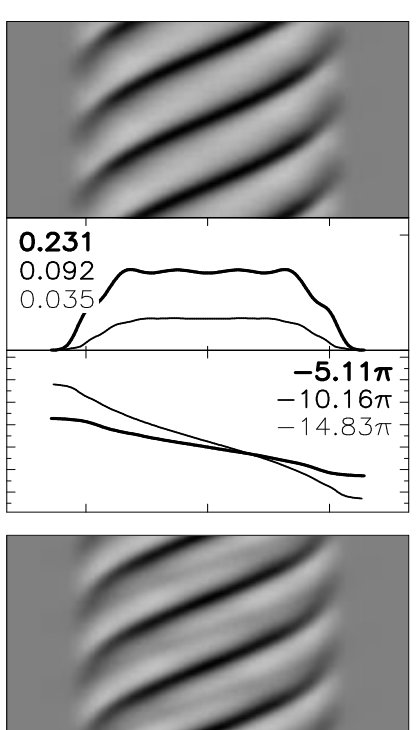

0.170
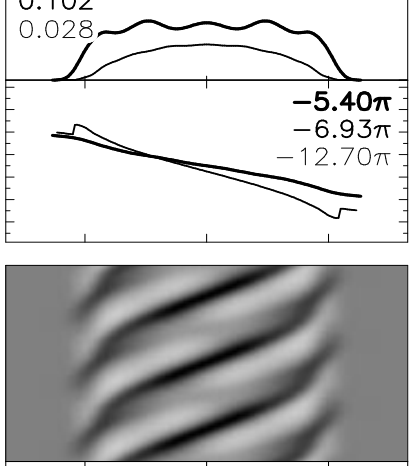

0.155

0.090

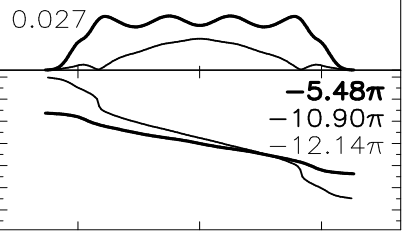

$i=30$
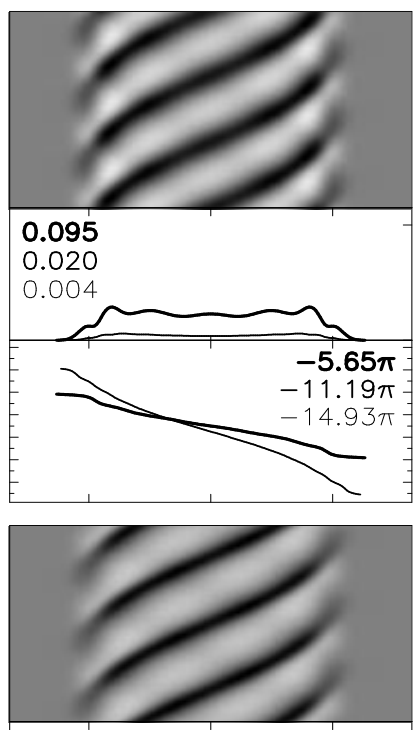

\subsection{1}
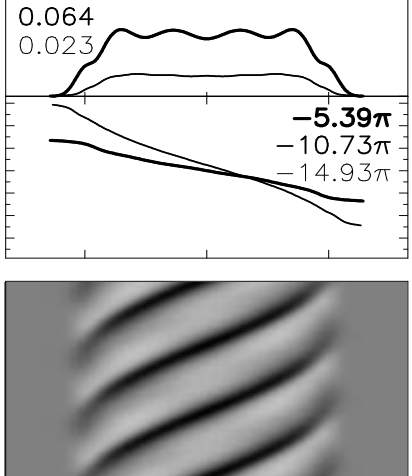

0.193

0.083
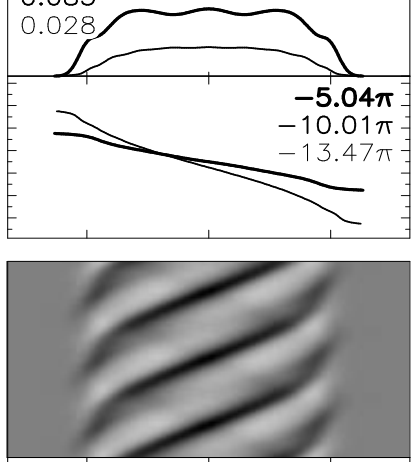

0.178

0.093

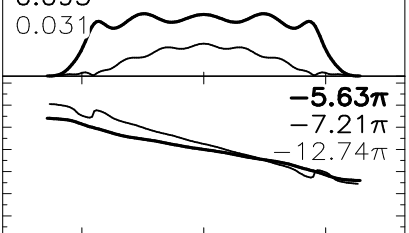

Fig. 5. b) Residual spectra, amplitude distribution and phase distribution of the time series of line profiles shown in Fig. 5 a. One of the things shown here is the canceling of line-profile variability for modes with $\ell-m$ an odd number, that are observed at an inclination of nearly $90^{\circ}$. Another feature seen in this figure is the fact that line-profile variations are not necessarily at largest for inclinations around $90^{\circ}$ (Sect. 6.1.1). Opposite to sectoral modes, the amplitudes of line-profile variations from tesseral modes generally tend to increase for decreasing inclinations. This illustrates that tesseral modes are equally well capable of producing large moving bumps, if the star is sufficiently inclined. We find that this behavior is not affected by any other parameter. Also note the wiggles in the amplitude distribution, which are commonly, but not exclusively, found for low inclination profiles 

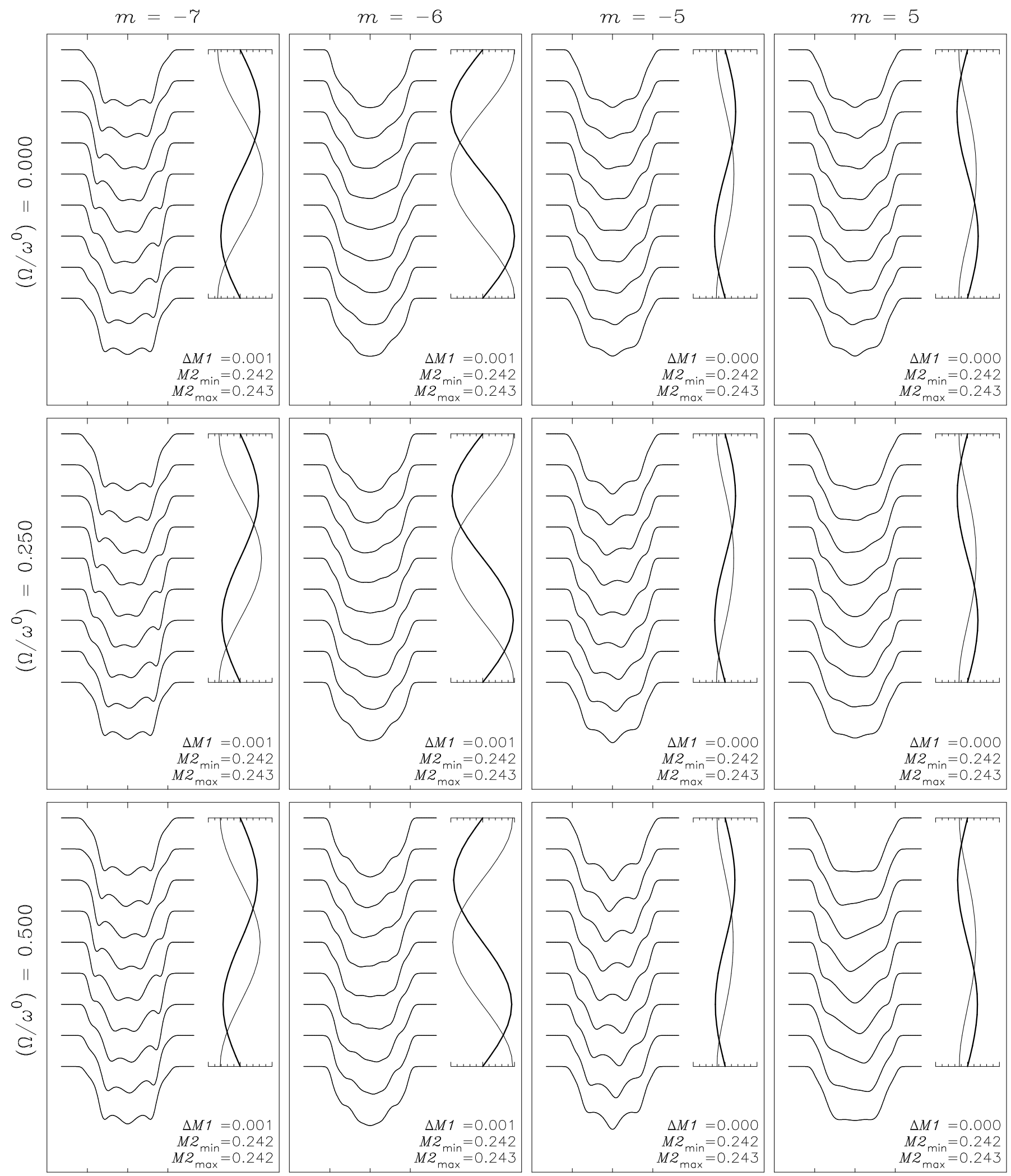

Fig. 6. a) Same as Fig. 4a but for $m$ against relative rotation rate $\Omega / \omega^{(0)}$. Fixed parameters are: $\ell=7, i=70^{\circ}, W=0.10 V_{\mathrm{e}}$ sin $i$, $V_{\max }=0.10 V_{\mathrm{e}} \sin i, k^{(0)}=5.0$ 

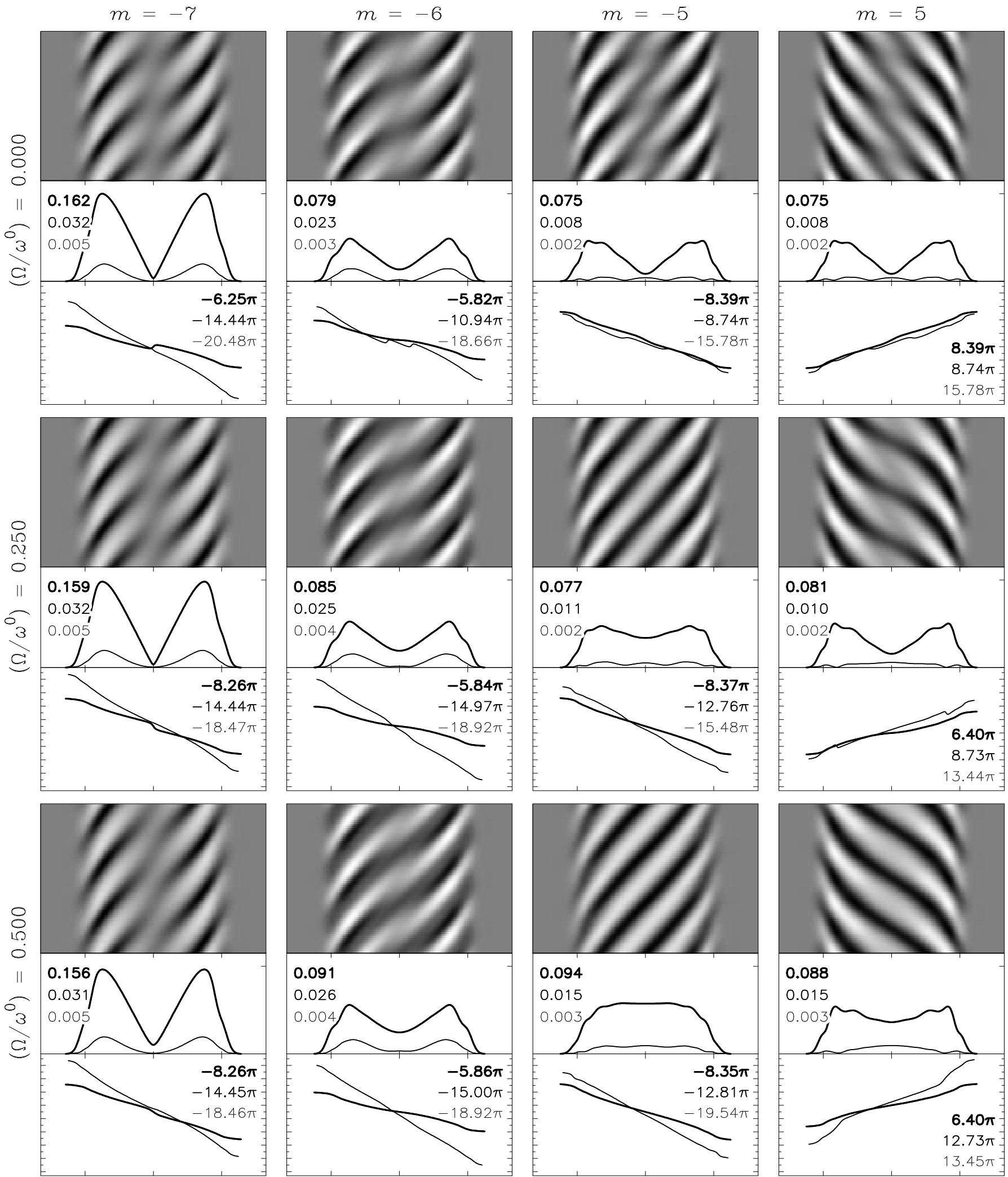

Fig. 6. b) Residual spectra, amplitude distribution and phase distribution of the time series of line profiles displayed in Fig. 6 a. This figure shows that the toroidal term due to the Coriolis force hardly affects the line profiles of high-degree sectoral modes. On the contrary, tesseral modes are affected. Extensive calculations, of tesseral modes at the highest rotation rate allowed in our model, revealed that the cases with $\ell-m$ an even number show the zero-rotation characteristics of a low $k^{(0)}$-value. This is best illustrated by the amplitude distributions in the column with $m=-5$. Also illustrated here (rightmost 2 columns) is the breaking of the symmetry between prograde and retrograde modes, when effects of rotation become important. Furthermore, the right column is an illustration of the $2 \pi$ phase jump in $\Delta \Psi_{0}$, that can occur when another source of variability in the line center (in this case toroidal movements) dominates the effect of radial motions (see Sect 6.1.2 and Fig. 9) 

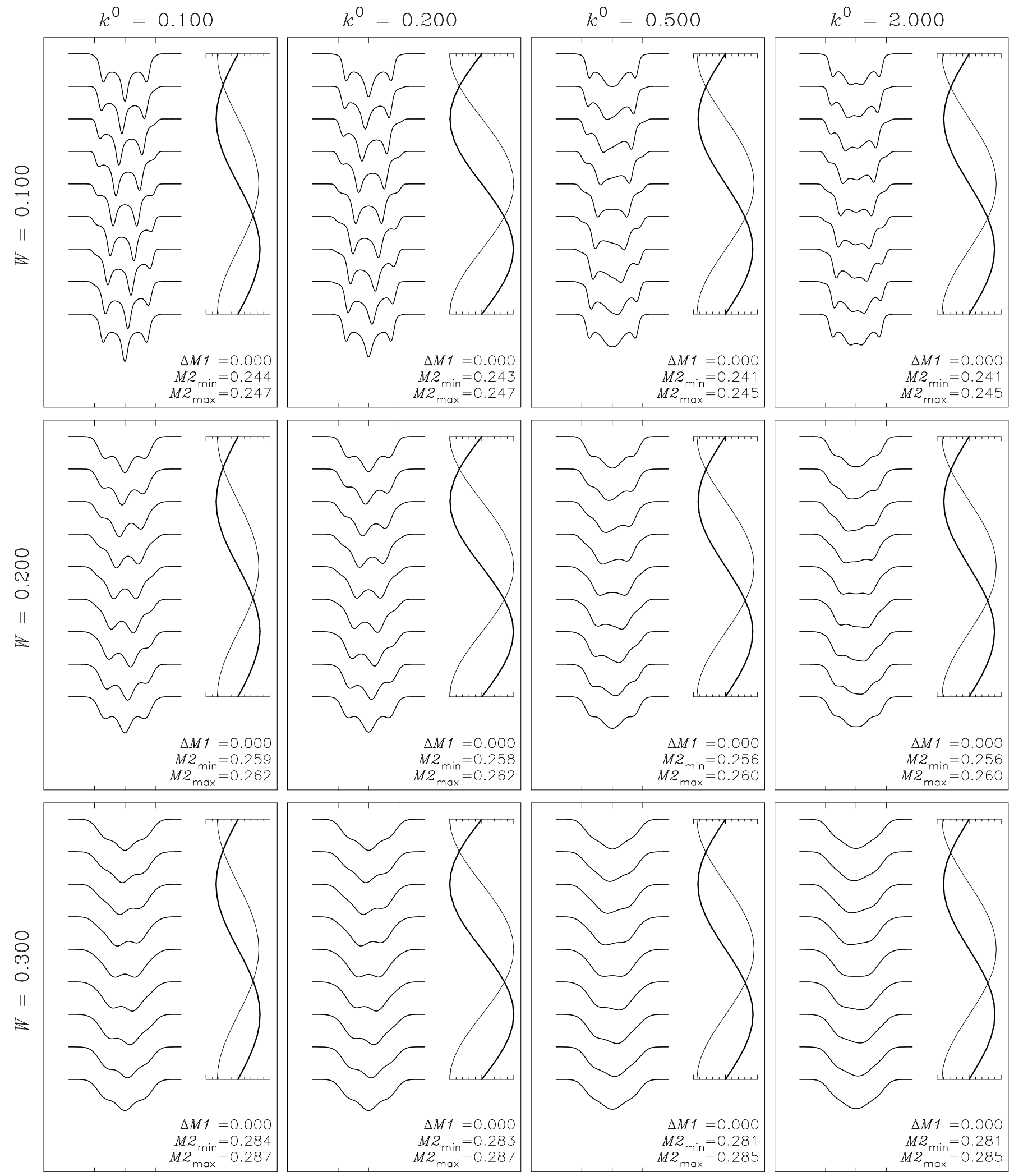

Fig. 7. a) Same as Fig. 4a but for intrinsic line-profile width $W$ (expressed in units of $V_{\mathrm{e}} \sin i$ ) against the ratio of the horizontal to the vertical velocity amplitudes $k^{(0)}$. Fixed parameters are: $\ell=8, m=-8, i=90^{\circ}, \Omega / \omega^{(0)}=0.15, V_{\max }=0.15 V_{\mathrm{e}} \sin i$. From the right column it can be seen that, for high values of $k^{(0)}$, the bumps that travel from blue to red through the line profile tend to disappear at the blue side of the line center, and reappear at the red side. This behavior of high $k^{(0)}$, low $W$ profiles disappears for the larger values of the intrinsic profile width $W$ 

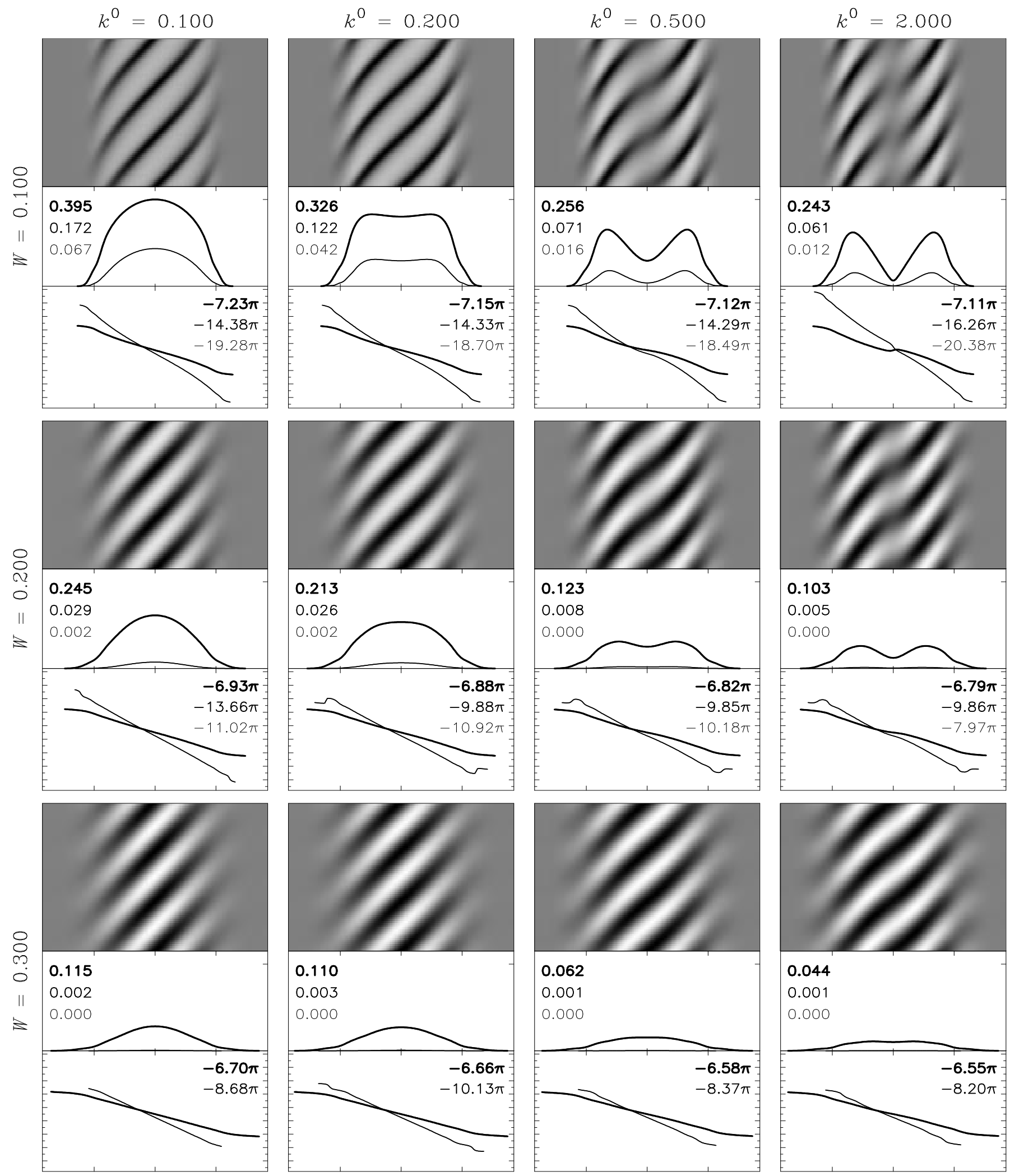

Fig. 7. b) Residual spectra, amplitude distribution and phase distribution of the time series of line profiles displayed in Fig. 7a. The figure shows the blurring of the line features for an increasing width of the intrinsic profile. The change in contrast of the bumps with $W$ is most evident from the grey-scale residual plots. For large intrinsic profile widths $W$ and any value of $k$, the amplitude distribution always has a shape that was previously considered as characteristic for a lower $k$-value and more narrow intrinsic profile. Also, the relation between the blue-to-red harmonic phase difference and the value of $|m|$, is much less evident for the higher values of $W$ 

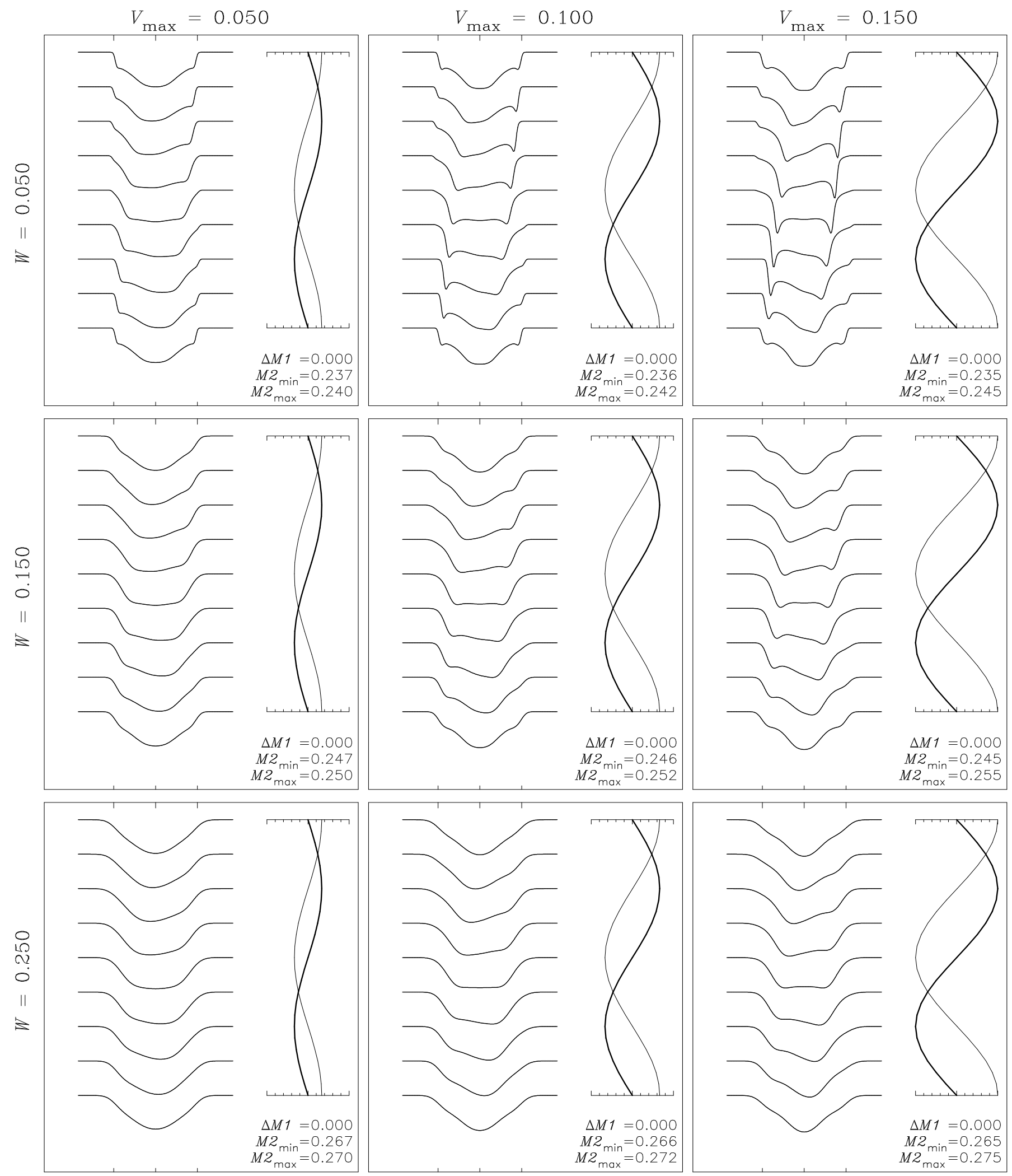

Fig. 8. a) Same as Fig. 4a but for velocity amplitude $V_{\max }$ against intrinsic line-profile width $W$ (both expressed in units of $\left.V_{\mathrm{e}} \sin i\right)$. Fixed parameters are: $\ell=6, m=-6, k^{(0)}=0.5, i=90^{\circ}, \Omega / \omega^{(0)}=0.10$. This figure clearly illustrates the increase of line-profile variability with increasing pulsation velocity and with decreasing intrinsic width. Also illustrated here, is that the variations of $M_{1}$ and $M_{2}$ are independent of $W$ 

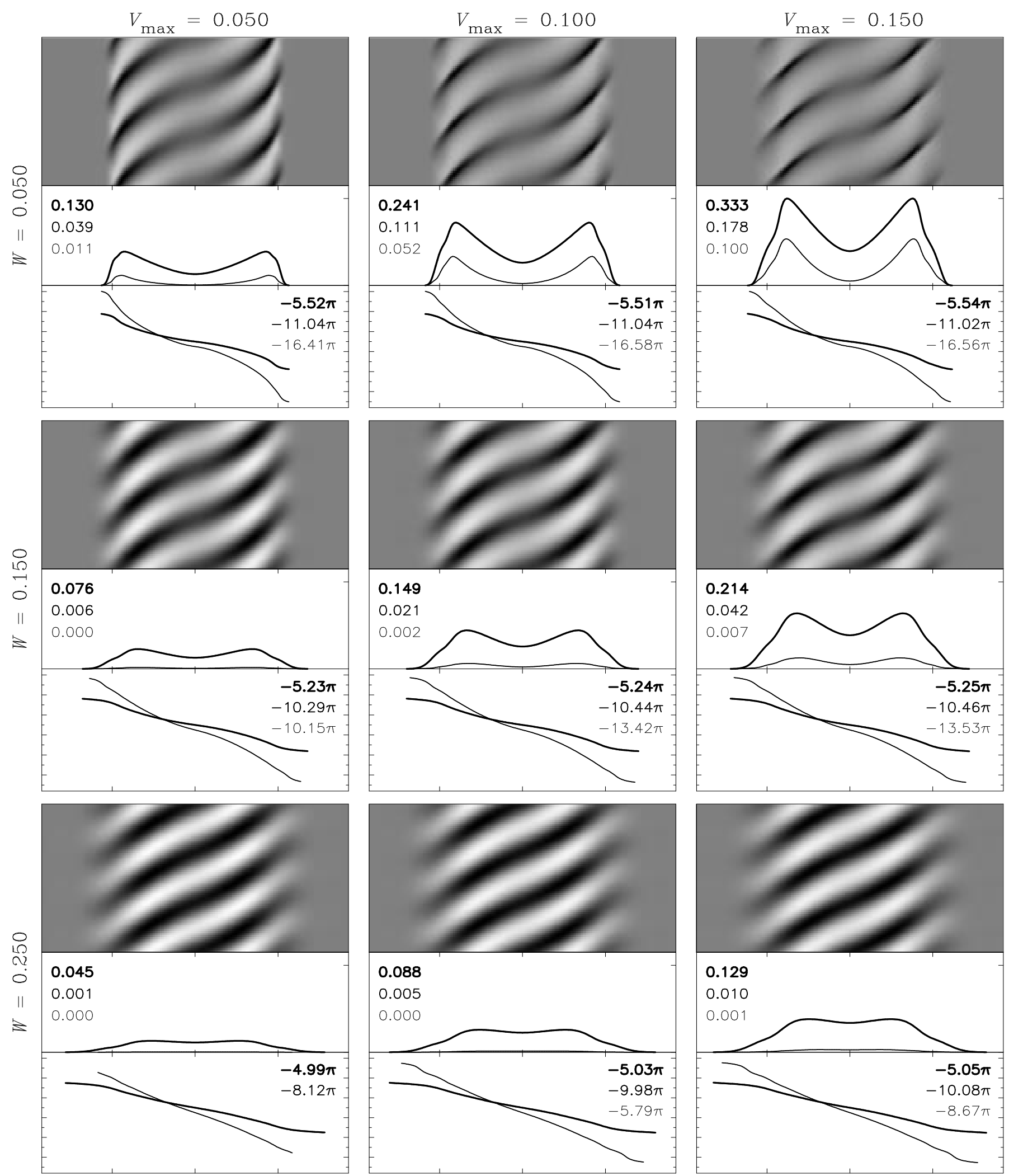

Fig. 8. b) Residual spectra, amplitude distribution and phase distribution of the time series of line profiles displayed in Fig. 8a. This figure shows the dependence of the maximum ratio of amplitudes $\left(I_{1} / I_{0}\right)_{\max }$ on the surface velocity amplitude $V_{\text {max }}$ and the intrinsic profile width $W$ (Sect.6.1.1). A higher value of $W$ or a lower value of $V_{\max }$ leads to more sinusoidal line-profile variability, i.e. a smaller value of $\left(I_{1} / I_{0}\right)_{\max }$ 

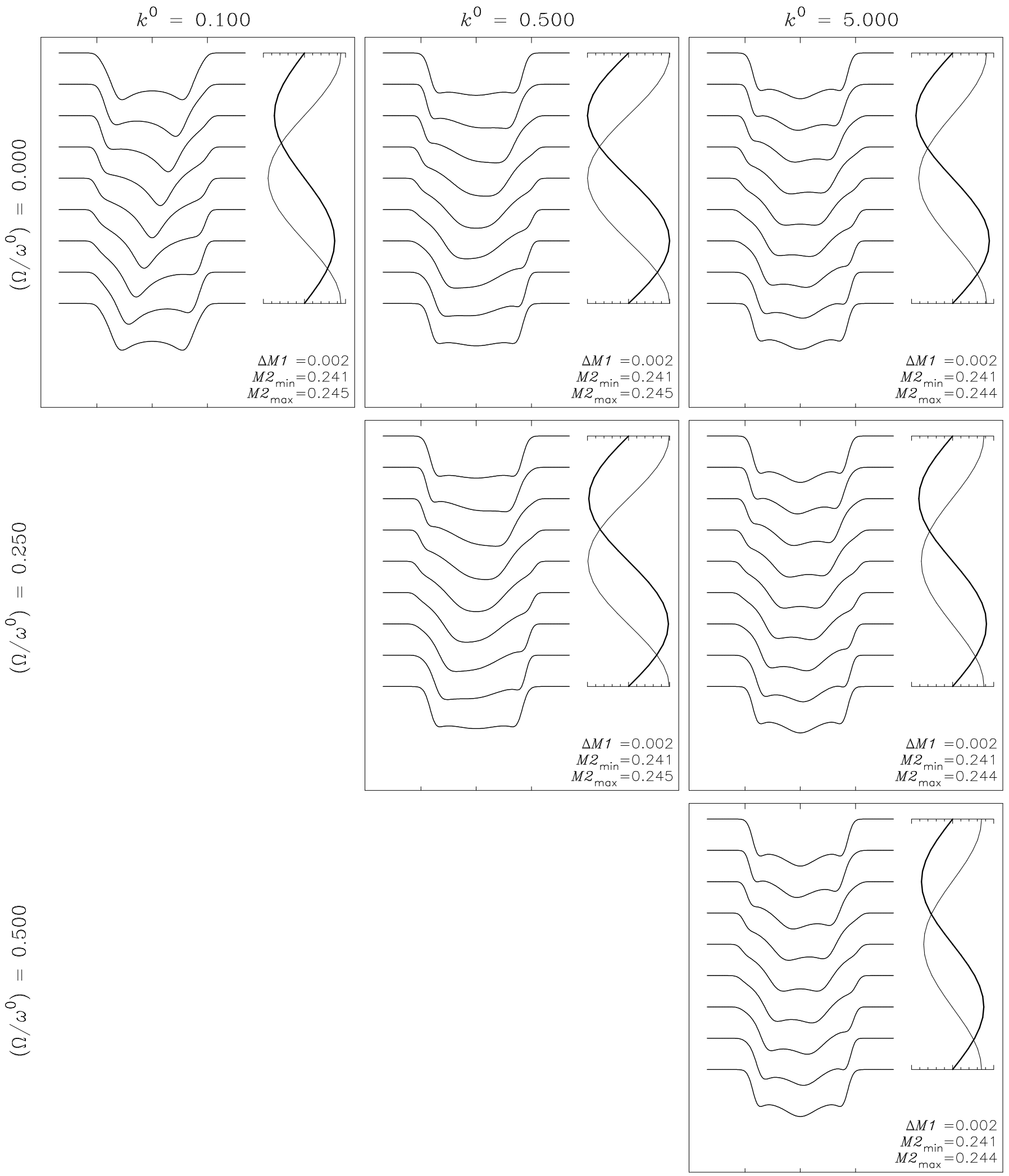

Fig. 9. a) Same as Fig. 4a but for the ratio of the horizontal to the vertical velocity amplitudes $k^{(0)}$ against the relative rotation rate $\Omega / \omega^{(0)}$. Fixed parameters are: $\ell=5, m=-5, i=75^{\circ}, W=0.10 V_{\mathrm{e}} \sin i, V_{\max }=0.10 V_{\mathrm{e}} \sin i$. The empty fields are for combinations of $k^{(0)}$ and $\Omega / \omega^{(0)}$ that imply an equatorial rotation velocity of more than $50 \%$ of break-up (see Sect. 5.1) 

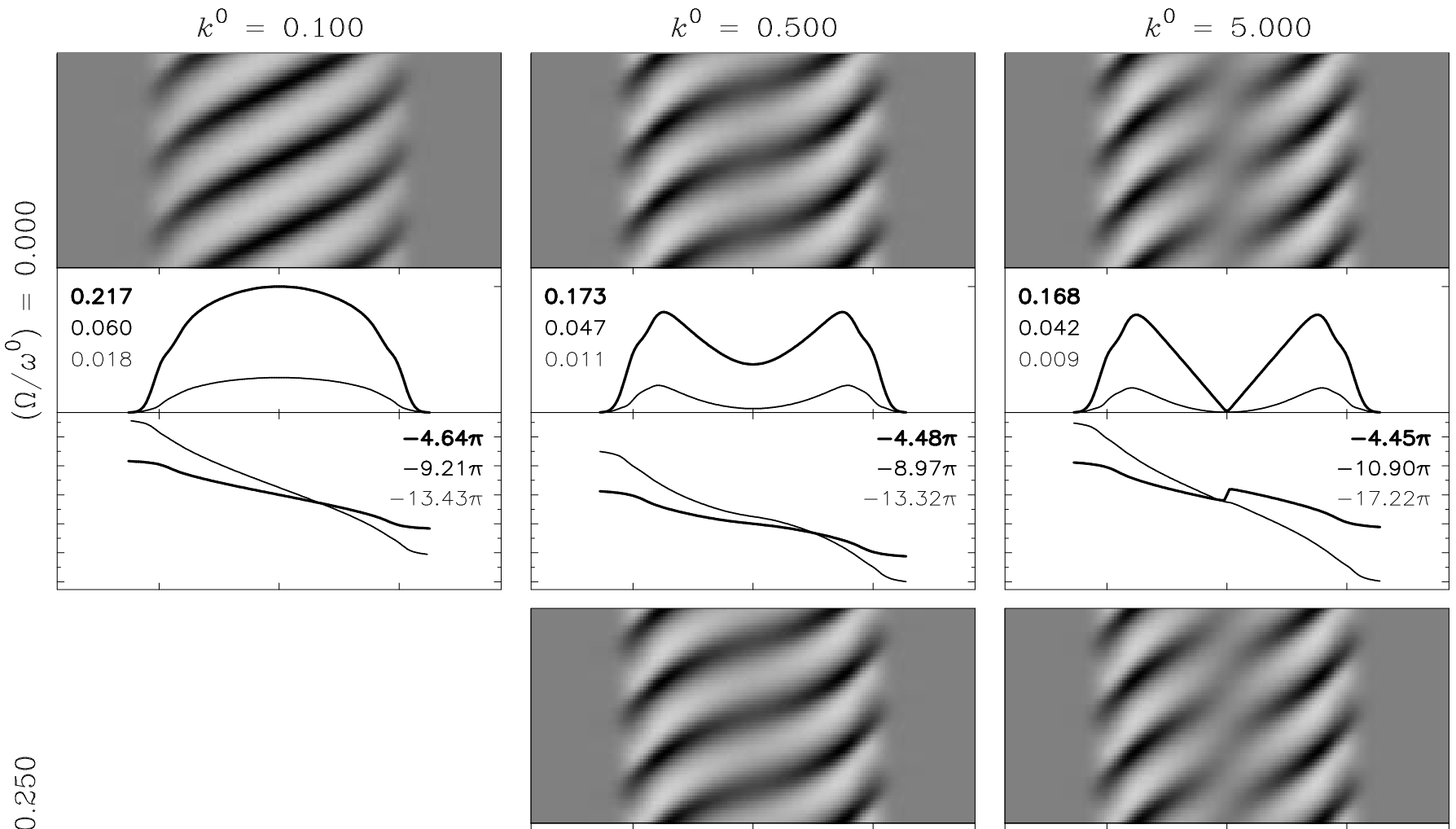

0
10
0
0
11
3
$\sqrt{3}$
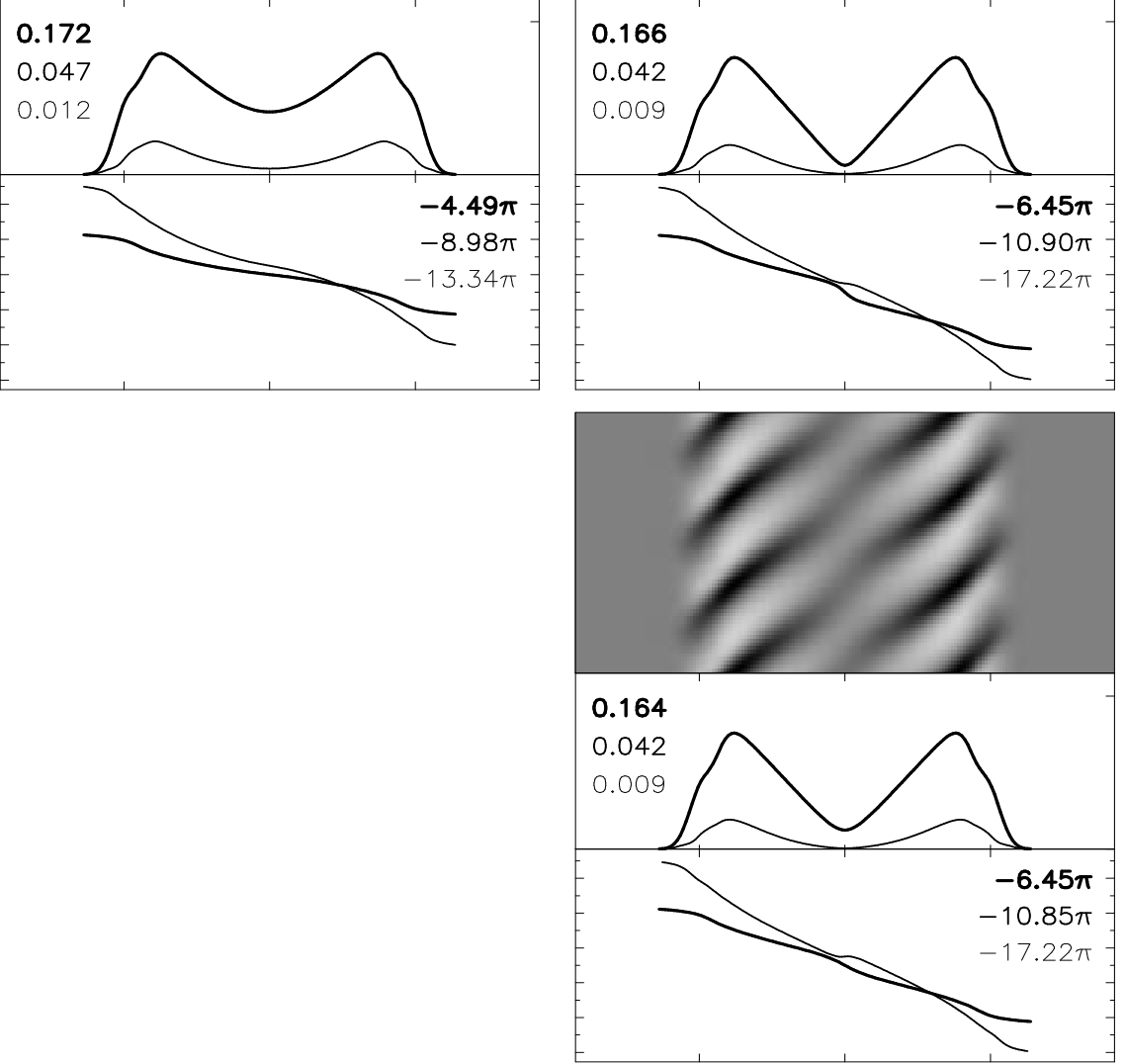

Fig. 9. b) Residual spectra, amplitude distribution and phase distribution of the time series of line profiles displayed in Fig. 9a. It is clear that for this pulsation mode the effects of rotation on the line-profile variability are very small. This figure also serves as an example of the $2 \pi$ jump in $\Delta \Psi_{0}$ (see Sect 6.1.2), which occurs for intermediate to high $k$-values, if additional variability (in this case the toroidal motion induced by the Coriolis force) is present at the line center. An additional source of variability at the line center can reconnect a bump at the blue side to a different one at the red side, leading to a $2 \pi$ jump in the blue-to-red phase difference 
Acknowledgements. We are grateful to Ilse De Boeck for the use of her computer code, which determines the $k$-values by means of numerical integration of the system of equations describing the pulsations up to first order in the rotation frequency, prior to publication. We thank Jan van Paradijs for his supportive remarks and his careful reading of the manuscript. CA is grateful to Dr. Alex Fullerton for initializing her into the field of Doppler Imaging and CLEAN. This research is supported by the Netherlands Foundation for Research in Astronomy (NFRA) with financial aid from the Netherlands Organization for Scientific Research (NWO), under project 781-71-043 (JHT).

\section{References}

Aerts C., De Pauw M., Waelkens C., 1992, A\&A 266, 294

Aerts C., 1993, Ph D Thesis, KU Leuven, Belgium

Aerts C., Waelkens C., 1993, A\&A 273, 135

Aerts C., Waelkens C., 1995, A\&A 293, 978

Baade D., 1984, A\&A 135, 101

Baade D. 1987, in: IAU Colloquium 92, Physics of Be stars, Slettebak A. and Snow T.P. (eds.). Cambridge University Press, p. 361

Balona L.A., 1986, MNRAS 219, 111

Carroll B.W., Hansen C.J., 1982, ApJ 263, 352

Clement M.J., 1994, in Pulsation, rotation and mass loss in early-type stars, Balona L.A., Henrichs H.F., Le Contel J.M. (eds.). Kluwer Academic Publisher, Dordrecht, p. 117 De Pauw M., Aerts C., Waelkens C., 1993, A\&A 280, 493

Gies D.R., Kullavanijaya A., 1988, ApJ 326, 813

Gies D.R. 1991, in Rapid Variability of OB stars, ESO Proc. 36, Baade D. (ed.), p. 299

Gies D.R.,1994, in Pulsation, rotation and mass loss in earlytype stars, Balona L.A., Henrichs H.F., Le Contel J.M. (eds.). Kluwer Academic Publisher, Dordrecht, p. 89
Hansen C.J., Cox J.P., Carroll B.W., 1978, ApJ 226, 210

Kambe E., Osaki Y., 1988, PASJ 40, 313

Kambe E., Ando H., Hirata R., 1990, PASJ 42, 687

Kennelly E.J., Walker G.A.H., Merryfield W.J., 1992, ApJ 400, L71

Ledoux P., 1951, ApJ 114, 373

Lee U., Saio H., 1990, ApJ 349, 570

Lee U., Jeffery C.S., Saio H., 1992, MNRAS 254, 185

Martens L., Smeyers P., 1982, A\&A 106, 317

Merryfield W.J., Kennelly E.J. 1993, in: GONG 1992, Seismic Investigation of the Sun and Stars, ASP Conf. Ser. 42, 363

Osaki Y., 1971, PASJ 23, 485

Reid A.H.N., Bolton C.T., Crowe R.A., Fieldus M.S., Fullerton A.W., Gies D.R., Howarth I.D., McDavid D., Prinja R.K., Smith K.C., 1993, ApJ 417, 320

Reid A.H.N., Aerts C. 1993, A\&A 279, L25

Saio H., 1981, ApJ 244, 299

Schrijvers C., Telting J.H., 1996, (Paper IV), (in preparation) Simon K.P., 1991, in Rapid Variability of OB stars, ESO Proc. 36, Baade D. (ed.), p. 335

Smith M.A., 1977, ApJ 215, 574

Smith M.A., 1986, in Hydrodynamic and magnetohydrodynamic problems in the sun and stars, Osaki Y. (ed.). University of Tokyo, Tokyo, p. 145.

Telting J.H., Schrijvers C., 1995, Proc IAU Symp 176 Vienna, Strassmeier K. (ed.), p. 35.

Telting J.H., Schrijvers C., 1996a, (Paper II), (in press)

Telting J.H., Schrijvers C., 1996b, (Paper III), (in press)

Townsend R.H.D., 1996, (in press)

Unno et al., 1989, Nonradial oscillations of stars, $2^{\text {nd }}$ edition. University of Tokyo Press, Tokyo

Vogt S.S., Penrod G.D., 1983, ApJ 275, 661

Vogt S.S., Penrod G.D., Hatzes A.P., 1987, ApJ 496, 127

Wade R.A., Rucinski S.M., 1985, A\&AS 60, 471

Yang S., Ninkov Z., Walker G.A.H., 1988, PASP 100, 233 